
\title{
$\begin{array}{ll}\text { Research Square } & \text { Preprints are preliminary reports that have not undergone peer review. } \\ \text { They should not be considered conclusive, used to inform clinical practice, } \\ \text { or referenced by the media as validated information. }\end{array}$
}

\section{Shared Genetic Architecture Between Alzheimer's Disease and Gastrointestinal Tract Disorders: A Large-scale Genome-wide Cross-trait Analysis}

\author{
Emmanuel 0 Adewuyi ( $\nabla$ e.adewuyi@ecu.edu.au ) \\ Edith Cowan University - Joondalup Campus: Edith Cowan University https://orcid.org/0000-0002-4533-0340
}

Eleanor K O’Brien

Edith Cowan University - Joondalup Campus: Edith Cowan University

\section{Dale R Nyholt}

Queensland University of Technology Statistical and Genomic Epidemiology Laboratory: Queensland University of Technology Institute of Health and Biomedical Innovation

Tenielle Porter

Edith Cowan University - Joondalup Campus: Edith Cowan University

Simon M Laws

Edith Cowan University - Joondalup Campus: Edith Cowan University

\section{Research article}

Keywords: Alzheimer's disease, Biological pathways, Causality, Gastro-intestinal tract disorders, Genetic overlap, Polygenicity, GWAS, Shared genetic susceptibility

Posted Date: November 11th, 2021

DOI: https://doi.org/10.21203/rs.3.rs-757229/v2

License: () (1) This work is licensed under a Creative Commons Attribution 4.0 International License. Read Full License

Version of Record: A version of this preprint was published at Communications Biology on July 18th, 2022. See the published version at https://doi.org/10.1038/s42003-022-03607-2. 


\section{Abstract}

Background: Consistent with the concept of the gut-brain phenomenon, observational studies have reported a pattern of co-occurring relationship between Alzheimer's disease (AD) and a range of gastrointestinal tract (GIT) traits. However, it is not clear whether the reported association reflects a causal or shared genetic aetiology of GIT disorders with AD. While AD has no known curative treatments, and its pathogenesis is not clearly understood, a comprehensive assessment of its shared genetics with diseases (comorbidities) can provide a deeper understanding of its underlying biological mechanisms and enhance potential therapy development.

Methods: We analysed large-scale genome-wide association studies (GWAS) summary data (sample size $=34,652-456,327$ ) to comprehensively assess shared genetic overlap and causality of GIT disorders with the risk of AD. Further, we performed meta-analyses, pairwise GWAS analysis; and investigated genes and biological pathways shared by AD and GIT disorders.

Results: Our analyses reveal significant concordance of SNP risk effects across AD and GIT disorders $\left(P_{\text {permuted }}=9.99 \times 10^{-4}\right)$. Also, we found a significant positive genetic correlation between $A D$ and each of gastroesophageal reflux disease (GERD), peptic ulcer disease (PUD), medications for GERD or PUD (PGM), gastritis-duodenitis, irritable bowel syndrome, and diverticular disease, but not inflammatory bowel disease. Mendelian randomisation analyses found no evidence for a significant causal association between AD and GIT disorders. However, shared independent genome-wide significant $\left(P_{\text {meta-analysis }}<5 \times 10^{-}\right.$ ${ }^{8}$ ) loci (including 1p31.3 [near gene, PDE4B], 1q32.2 [CD46], 3p21.31 [SEMA3F], 16q22.1 [MTSS2], 17q21.33 [PHB], and 19q13.32 [APOE]) were identified for AD and PGM, six of which are putatively novel. These loci were replicated using GERD and PUD GWAS and reinforced in pairwise GWAS (colocalisation) as well as gene-based analyses. Lipid metabolism, autoimmune system, lipase inhibitors, PD-1 signalling, and statin mechanisms were significantly enriched in pathway-based analyses.

Conclusions: These findings support shared genetic susceptibility of GIT disorders with AD risk and provide new insights into their observed association. The identified loci and genes-PDE4B, CD46 and $A P O E$, especially-and biological pathways-statins and lipase inhibitors, in particular-may provide novel therapeutic avenues or targets for further investigation in AD, GIT disorders, or their comorbidity.

\section{Background}

Alzheimer's disease (AD) is the most prevalent form of dementia, characterised by neurodegeneration and a progressive decline in cognitive ability beyond what would be expected from the normal ageing process [1, 2]. The disorder ranks as a subject of significant global public health importance with consequences for wide-ranging social and economic adverse impacts on sufferers, their families, and the society at large [1]. By the year 2030, over 82 million people-and about 152 million by 2050-are projected to suffer from AD [1,2]. The annual global economic costs of the disorder are currently estimated at nearly one trillion US dollars and predicted to reach two trillion US dollars in 2030 [1-4]. While AD has no known curative treatments, and its pathogenesis is yet to be clearly understood, a comprehensive assessment of its shared genetics with other diseases (comorbidities) can provide a deeper understanding of its underlying biological mechanisms and enhance potential therapy development.

Several studies have reported a pattern of co-occurrence of dementia (and AD in particular) with certain gastrointestinal tract (GIT) disorders, microbiota, dysbiosis or medications commonly used in the treatment of peptic ulcer disease (PUD) [5-12]. For example, an observational study reported more than twice the odds of dementia in individuals with gastritis (adjusted odds ratio [AOR]: 2.42, $\mathrm{P}<0.001,95 \%$ confidence interval [CI]: $1.68-3.49$ ) [5]. Another observational study found a significant association between regular use of proton-pump inhibitors (PPI, medications for gastritis-duodenitis, gastroesophageal reflux disease [GERD] or PUD) and increased risk of incident dementia (hazard ratio [HR]: 1.44 [95\% Cl, 1.36 - 1.52]; P < 0.001) [6]. Similarly, lansoprazole (a PPI) was reported to promote amyloid-beta (Ab) production [7], the accumulation of which is central to one of the core hypotheses for the development of $A D$ [13]. More recently, a large population-based longitudinal study reported a more than a six-fold increased risk of $A D$ in individuals with inflammatory bowel disease (IBD) [HR: 6.19, 95\%Cl: 3.31 - 11.57], predicting over five-fold increased incidence across all forms of dementia [9]

Taken together, available evidence suggests comorbidity or some form of association between AD and GIT disorders, although it is not clear whether GIT traits are risk factors for AD or vice versa. Regardless, these findings agree with the concept of the 'gut-brain' axis or the 'gastric mucosa-brain' relationships, which has been implicated in the association between GIT-related traits and central nervous system (CNS) disorders including depression and Parkinson's disease [14-19]. In support of a possible link between AD and GIT traits, a recent animal model-based study indicates that intra-gastrointestinal accumulation of Ab may induce gastric function alteration, CNS amyloidosis, and subsequent AD-like dementia [20]. Comorbidity of AD and GIT disorders may worsen the quality of life of sufferers while contributing significantly to increased healthcare costs.

Despite the increasing number of studies reporting an association between AD and GIT traits, the biological mechanism(s) underlying this potential association remains unclear. Moreover, contrasting evidence exists [9, 21, 22], and many questions are unanswered. First, is there a risk-increasing association between AD and GIT disorders (including medicines commonly used for PUD, GERD, or gastritis-duodenitis)? This question assumes great importance in the face of contrasting evidence and longstanding debates on the potential roles of GIT traits in the risk of AD [18, 21-23]. Second, is there a cause-and-effect relationship between AD and GIT disorders (vertical pleiotropy)? Third, are there genetic components-e.g., single nucleotide polymorphism (SNPs), genes, and genomic loci-shared by AD and GIT disorders (biological pleiotropy)? Last, what biological pathways, processes, or mechanisms underlie comorbidity or any association between $A D$ and GIT disorders?

Large-scale genome-wide association studies (GWAS), identifying an increasing number of SNPs, genes, and susceptibility loci, have been conducted separately for $A D$ and a range of GIT traits [24-27]. Findings from these GWAS provide compelling evidence for the roles of genetics in the aetiologies of $A D$ and GIT disorders including PUD, medications for PUD or GERD (PGM), gastritis-duodenitis, GERD, irritable bowel syndrome (IBS), diverticular disease, and 
IBD [24-27]. However, to the best of our knowledge, no study has leveraged the possible pleiotropy between AD and GIT disorders as a basis for discovering new shared SNPs, genes and/or susceptibility loci. Thus, it is unclear whether AD shares genetic aetiology with any of these GIT disorders.

Moreover, studies assessing the mechanism(s) of association between AD and GIT disorders, based on the analysis of molecular genetic data, are lacking. We use a set of statistical genetics approaches in the analysis of well-powered GWAS data to comprehensively assess the genetic relationship between AD and GIT disorders-PUD, GERD, PGM, IBS, gastritis-duodenitis, diverticular disease, and IBD. The outcomes of this study have the potential to improve our understanding of the genetic architecture of $A D$ and each of the GIT disorders, provide insights into their possible underlying biology, and characterise potential targets for further investigation in their mechanisms or therapy development.

\section{Methods}

Fig. 1 presents a schematic workflow and design for this study. Briefly, we performed three broad levels of analyses-SNP-level, gene-level, and pathway-based analysis-to comprehensively assess the genetic relationships between AD and GIT disorders. In each of the levels, we analysed well-powered GWAS data using a set of well-regarded statistical genetics methods. First, we used the 'SNP effect concordance analysis' (SECA) [28] method for SNP-level genetic overlap assessment and the linkage disequilibrium score regression (LDSC) [29] method for genetic correlation analysis between AD and each of the GIT traits. Second, to identify SNPs and susceptibility loci shared by AD and GIT disorders, we carried out GWAS meta-analysis using several complementary models, leveraging the increased power from data pooling and pleiotropy of genetic variants. We also applied the pairwise GWAS (colocalisation) method [30] to identify independent genomic loci with shared genetic influence on AD and GIT disorders. Third, using the Mendelian randomisation (MR) [31] and the Latent Causal Variable (LCV) [32] methods, we investigated potential causal (or partial causal [LCV]) associations between AD and each of the GIT disorders. Fourth, we performed gene-based association analysis to identify genes shared by AD and GIT disorders reaching genome-wide significance. Lastly, we used pathway-based analysis to identify potential biological mechanisms shared by AD and GIT disorders.

\section{GWAS summary statistics}

The GWAS data utilised in the present study are summarised in Table 1 with further cohort-specific details provided in Additional file 1: Table S1. The data were sourced from popular GWAS databases, repositories, and large research consortia/groups. The GWAS summary data for 'clinically diagnosed AD and AD-by-proxy' [24] was used as our AD GWAS data. This GWAS has large sample size (cases $=71,880$, controls $=383,378$, sample size $[\mathrm{N}]=455,258$ ) and, thus, increased power for detecting genetic variants of small to moderate effect sizes. More specific details about the data have been published [24]. GIT traits including PUD (cases $=16,666$, controls $=439,661, \mathrm{~N}=456,327$ ), IBS (cases $=28,518$, controls $=426,803, \mathrm{~N}=455,321)$, and IBD (cases $=7,045$, controls $=$ $426803, N=456,327$ ) were assessed against AD. The GWAS for the traits were obtained through the recently published GIT GWAS [25] and other sources located through the GWAS Atlas [27]. Clinically, PUD medications are indicated in GERD, accordingly, GWAS for PUD and GERD medications have been conducted [25]. This GWAS has a large sample size (cases $=90,175$, controls $=366,152, N=456,327$ ), and as was the case in the original publication [25], we utilised the data for analysis in the present study. These GIT GWAS were well characterised and, where possible, validated as described in the original publication [25].

Additionally, we utilised a well-characterised GWAS for GERD (cases $=71,522$, controls $=261,079, \mathrm{~N}=332,601$ ), which combined datasets from the UK Biobank and the QSKIN study [26]. Gastritis-duodenitis (cases $=28,941$, controls $=378,124, \mathrm{~N}=407,065)$ and diverticular disease $($ cases $=27,311$, controls $=$ $334,783, N=362,094$ ) GWAS from the Lee Lab (https://www.leelabsg.org/resources) were also used in this study. A comprehensive description of the quality control procedures for each of the GWAS data and their analysis are available through the corresponding publications (Additional file 1: Table S1). Notably, our preliminary analysis indicates that there is no significant sample overlap between the AD GWAS and each of the GIT phenotypes assessed in this study, ruling out potential bias from such occurrence.

\section{SNP effect concordance analysis (SECA)}

We used the standalone version of the SECA software pipeline (https://sites.google.com/site/qutsgel/software/seca-local-version) to perform SNP-level genetic overlap assessment and statistical tests between AD and GIT disorders. A detailed description of the SECA software and methods has been published [28]. Briefly, SECA accepts a pair of GWAS data (dataset 1 and dataset 2) as input and performs a range of analyses to determine whether there is genetic overlap (shared genetics) between a pair of traits-AD and GIT disorders in the present study. First, we carried out quality control to exclude all nonrsID(s) and duplicate variants in dataset 1, align SNP effects to the same effect allele across dataset 1 and dataset 2 and perform a P-value informed test for linkage disequilibrium (LD) clumping in the dataset 1 using PLINK [33].

Second, SECA partitions independent SNPs resulting from LD clumping (at $r^{2}<0.1$ ) into 12 subsets of SNPs according to the P-value for dataset 1 as follows: $\mathrm{P} 1 \leq(0.01,0.05,0.1,0.2,0.3,0.4,0.5,0.6,0.7,0.8,0.9,1.0)$. SECA subsequently performs Fisher's exact tests (FT) to assess the presence of excess SNPs in which the direction of effects is concordant across dataset 1 and dataset 2 (that is, for the corresponding P-value derived 12 subsets of SNPs associated in dataset 2, P2). Hence, a total of 144 SNP subsets (a 12 by 12 matrix from dataset 1 and dataset 2) was assessed for SNP effect concordance. In the present study, we first assessed AD GWAS as dataset 1 and each of the GIT disorders as dataset 2. For comparison, we also assessed each of the GIT disorders as dataset 1 against AD as dataset 2. Thus, using SECA, we assessed the effects of AD-associated SNPs on each of the GIT disorders and vice versa. This is an important analysis step to account for instances where SNPs that are strongly associated with AD do not affect GIT traits and vice versa. 
LDSC assesses and distinguishes the contributions of polygenicity, sample overlaps, and population stratification to the heritability and genetic correlation between traits [29]. In the present study, we performed LDSC analysis using the standalone version of the software and by following the procedures provided by the program developer (https://github.com/bulik/ldsc). We conducted cross-trait bivariate LDSC to estimate the genetic correlation between AD and each of the GIT disorders assessed in this study. To avoid a potential bias from residual confounding, we did not constrain the genetic covariance intercepts in any of our genetic correlation analyses; this practice may be conservative and reduce the significance of correlation estimates in instances where there is no sample overlap between the pair of traits being assessed.

\section{GWAS Cross-traits meta-analysis}

GWAS meta-analysis pools the results of GWAS data, thereby increasing the sample sizes and augmenting the detection of genetic variants with small to modest effect sizes. In the present study, we used the GWAS meta-analysis method of pooling AD GWAS with each of the GIT traits (cross-disorder or crosstrait meta-analysis). We used two models of meta-analysis: the Fixed Effect (FE), and the modified Random Effect (RE2) [34] models. The FE model estimates the FE P-value using the inverse-variance weighted method, which assumes that the AD and each of the GIT disorders' GWAS are assessing the same (fixed) effect. The presence of effect heterogeneity is a limitation of the model. On the other hand, by estimating P-values using the modified random-effects, the RE2 model [34] allows for differences in SNP effects and the method is powerful in the presence of SNP effect heterogeneity. Furthermore, given the possibility of negligible sample overlap between AD and GERD GWAS (genetic covariance intercept $=0.0133$, se $=0.005$ ) and AD and PGM GWAS (genetic covariance intercept $=0.0135$, se $=0.006$ ), we utilised the modified random-effects correlation (RE2C) [35] as an additional model of meta-analysis. This method is suitable for GWAS meta-analysis of correlated statistics [35].

\section{Genomic loci characterisation}

Using the outputs of our cross-trait meta-analyses for AD and each of the GIT disorders, we carried out some downstream analyses including functional annotation of SNPS, and genomic loci characterisation in line with practice in previous studies [15, 36, 37]. Briefly, SNPs that were not genome-wide significant in the individual $A D$ and GIT disorder GWAS, but which reached genome-wide significance following the meta-analysis were identified. From these, we characterised independent SNPs at $r^{2}<0.6$, and lead SNPs at $r^{2}<0.1$. We defined the genomic locus as the region within $250 \mathrm{~kb}$ of each lead SNP. We assigned lead SNPs within this region to the same locus, meaning two or more lead SNPs may be present in one locus. Several of these downstream analyses were performed in the Functional Mapping and Annotation (FUMA) software (an online platform) [36].

\section{Assessment using the posterior probability (m-value) method}

To identify loci shared by AD and GIT disorders, we performed a further analysis using the posterior probability (m-value) method and the complementary binary effects (BE) P-value estimates [38]. Briefly, cross-study information was utilised in estimating the m-value to predict whether a SNP or locus has effects in each of the studies meta-analysed, particularly in the presence of effect heterogeneity [38]. M-value ranges from 0 to 1 , where a value $>0.9$ predicts that an effect exists for the SNP or locus in the study (e.g., AD GWAS or PUD GWAS). On the other hand, an m-value $<0.1$ predicts that an effect does not exist in the study. M-values from 0.1 to 0.9 predict an ambiguous effect. We used these methods to further assess whether the SNPs or loci identified in our GWAS metaanalysis have effects (shared) by the two traits under investigation, especially where the test for heterogeneity was significant. We interpreted the results of the m-value alongside the BE P-value. It is expected that, where effects exist in both traits being assessed, the BE P-value estimates will be less than the P-value for the respective GWAS. We implemented the BE and m-value methods alongside the FE, and the RE2 meta-analysis models.

\section{Pairwise GWAS analysis}

We performed co-localisation analysis utilising the pairwise GWAS (GWAS-PW) method [30] to further assess the regions in the genome shared by AD and GITdisorders. Briefly, GWAS-PW software implements the Bayesian pleiotropy association test and identifies genomic regions that influence a pair of correlated traits [30]. We used this method to assess whether the loci reaching genome-wide significance in our GWAS meta-analyses were truly shared by AD and the GIT disorders. Also, we investigated other shared genomic regions which may not have been found in the GWAS meta-analysis. We combined the summary data for AD with the data for each of the GIT disorders and estimated the posterior probability of association (PPA) of a genomic region using the GWAS-PW software. We modelled four PPAs: i) that a genomic region is associated with AD only (PPA-1), ii) that a genomic region is associated with the GIT trait only (PPA-2), iii) that a genomic region is associated with both $A D$ and the GIT trait (PPA-3), and iv) that a specific genomic region is associated with both $A D$ and the GIT trait but through separate causal variants (PPA-4) [30].

\section{Causal relationships assessment}

Using Mendelian randomisation (MR) [31] analysis methods, we test for a causal association between AD and each of the GIT disorders assessed in this study. Mimicking randomised control trials (RCTs), MR analysis incorporates genetics into epidemiological study designs to assess causality [31]. In the present study, we used the two-sample MR method (https://mrcieu.github.io/TwoSampleMR/articles/introduction.html) for a bidirectional association (as it is customarily done) assessment between $A D$ and each of the GIT disorders. In the first round of analysis (AD as exposure variable), independent $\left(r^{2}<0.001\right)$ genome-wide significant SNPs $\left(P<5 \times 10^{-8}\right)$ associated with AD were utilised as instrumental variables (IVs) and assessed against each of the GIT disorders' GWAS (outcome variables) analysed in this study. This analysis assesses whether genetic predisposition to AD is causally associated with any of the GIT traits included in the present study.

Reversing the direction of analysis, independent SNPs robustly associated with each of the GIT disorders' GWAS (exposure variable) were similarly utilised as IVs and assessed against AD (as the outcome variable). In this instance, we assessed the potential causal effects of GIT traits on AD. We used the inverse variance weighted (IVW) model of MR as the primary method for causal association assessment, and for validity testing, we performed a heterogeneity test (Cochran's Q-test), a 'leave-one-out' analysis, a horizontal pleiotropy check (MR-Egger intercept) and individual SNP MR analyses. Also, we used other MR 
analysis models including the weighted mode, simple mode, MR-Egger, weighted median [39, 40], and the 'Mendelian randomisation pleiotropy residual sum and outlier' (MR-PRESSO) [41] methods for sensitivity testing. All MR analyses were performed in R (4.0.2).

We performed an additional assessment of the causal or partial causal association between AD and each of the GIT disorders using the Latent Causal Variable (LCV) method [32]. LCV estimates causality proportion (GCP) ranging from -1 to 1 where a value close to 1 indicates a potential causal association between two traits in the forward direction and -1 in the backward direction [32]. LCV corrects for heritability and genetic correlation between traits and is not limited by sample overlap [32]. This analysis was performed in the online platform of the Genetics of Complex Traits (CTG) virtual laboratory (https://vl.genoma.io/analyses/lcv) [32, 42].

\section{Gene-based association analysis}

We performed gene-based association analyses to identify genome-wide significant genes shared by both AD and each of the GIT disorders assessed in this study. This analysis complements the SNP-based studies. However, beyond the SNP-level analysis, gene-based association analysis provides greater power for identifying genetic risk variants since it aggregates the effects of multiple SNPs, and it is generally not limited by small effect sizes or correlations among SNPs. Moreover, genes are more closely related to biology than SNPs, meaning gene-level analysis can offer better insights into the underlying biological mechanisms of complex traits.

In the present study, we carried out gene-based association analysis separately for AD and each of the GIT disorders using the Generalized Gene-Set Analysis of GWAS Data (MAGMA) software, implemented in FUMA (https://fuma.ctglab.nl/) [36]. Based on the results of the gene-based analysis, we identified genome-wide significant genes for each of the traits. Also, using the Fisher Combined P-value (FCP) method, we identified genes shared by AD and each of the GIT traits.

\section{Pathway-based functional enrichment analysis}

For a better understanding of the potential biological mechanisms underlying AD and GIT disorders or their co-occurrence, we carried out pathway-based functional enrichment analyses using the online platform of the g:GOst tool in the g-profiler software [43]. This analysis enables us to functionally interpret genes overlapping AD and GIT disorders. We included genes that were overlapping between $A D$ and each of GERD and PGM at $P_{\text {gene }}<0.05$ (FCP $<0.02$ ) in this analysis, and followed established protocols [44]. Functional category term sizes were restricted to values from 5 to 350 [44]. For multiple testing corrections, we applied the default ' $\mathrm{g}$ : SCS algorithm' recommended in the protocol [44] and reported the significantly enriched biological pathways at the multiple testing adjusted $P$-value $\left[P_{\text {adjusted }}\right]<0.05$.

\section{Results}

\section{Genetic overlap between AD and GIT disorders}

We first tested for SNP-level genetic overlap between AD and GIT disorders using the SNP effect concordance analysis (SECA) method [28]. Briefly, SECA performs a bi-directional analysis, assessing the effects of AD-associated SNPs (dataset 1) on each of the GIT disorders (dataset 2) and vice versa. We found a significant concordance of SNP risk effects between the AD GWAS and each of the GERD, PUD, PGM, gastritis-duodenitis, IBS and diverticular disease GWAS, indicating that there is a strong genetic overlap between $A D$ and each of these phenotypes. Table 2 summarises the results of the primary test for the concordance of effects in which 144 SNP subsets were tested, with AD as P1 (dataset 1) and GERD as P2 (dataset 2). All these SNP subsets showed significant concordance of effects (Odds ratio $[O R]>1$ and $P<0.05$ ) with a permuted $P$-value $\left(P_{\text {permuted }}\right)=9.99 \times 10^{-4}$. A total of 26,963 linkage disequilibrium (LD)-independent SNPs $\left(r^{2}<0.1\right)$ were common to both the AD and GERD GWAS (at P1 $\left.1_{\text {GWAS-data }}=\mathrm{P} 2_{\text {GWAS-data }} \leq 1\right), 13,955$ (52\%) of which exhibit significant effect concordance across the two GWAS $\left(\mathrm{OR}=1.18\right.$, $\left.P_{\text {Fisher'sexact }}=4.65 \times 10^{-11}\right)[$ Table 2].

As expected, a pattern of increasing strength of association between AD and GERD (measured using the OR values) was observed as the P-values for the SNP subsets (P1 and P2) decrease. For example, at AD (P1 $\left.1_{\text {GWAS-data }}<0.05\right)$ and GERD (P2 $\left.{ }_{\text {GWAS-data }}<0.05\right)$, the proportion of SNP effect concordance is $58 \%(0 R=$ $\left.1.84, P_{\text {Fisher's-exact }}=1.74 \times 10^{-6}\right)$, increasing to $61 \%$ at $\mathrm{P} 1=\mathrm{P} 2<0.01$. In a reverse analysis (GERD as dataset $1(\mathrm{P} 1)$ and $A D$ as dataset $\left.2(\mathrm{P} 2)\right)$ using $S E C A$, we also found all the 144 subsets of SNPs $\left(O R>1\right.$ and $\left.P<0.05, P_{\text {permuted }}=9.99 \times 10^{-4}\right)$ showing significant concordance of SNP risk effects across the two disorders [Additional file 2: Supplementary Note 1]. These results indicate that AD-associated SNPs are also associated with GERD, and vice versa-supporting evidence of highly significant genetic overlap between the two disorders.

SECA analyses also revealed a similar significant genetic overlap between AD and each of PGM, gastritis-duodenitis, diverticulosis and PUD (Table 3). While there was significant genetic overlap between AD and IBS, the strength of association was comparatively less than for the other GIT disorders (Additional file 2: Supplementary Note 1). For AD and IBD, SECA revealed significant concordance of SNP risk effects when AD was assessed (as dataset 1 ) against IBD (as dataset 2) $\left[O R>1\right.$ and $\left.P_{\text {GWAS-data }}<0.05, P_{\text {permuted }}=0.025\right]$ but not the other way around (Additional file 2: Supplementary Note 1). The observed significant overlap between $A D$ (dataset 1) and IBD (dataset 2) was much weaker than for the rest of the GIT disorders assessed. Table 3 summarises the results of our SECA-based genetic overlap assessment between AD and GIT traits.

\section{Genetic correlation between AD and GIT disorders}

We used the linkage disequilibrium score regression (LDSC) method to further assess and quantify the SNP-level genetic correlation between AD and GIT disorders. The apolipoprotein $\mathrm{E}(A P O E)$ region has a large effect on the risk of AD; hence, we excluded $A P O E$ and the 500 kilobase (kb) flanking region (hy19, 19:44,909,039 - 45,912,650) from our AD GWAS for this analysis. Given the complex LD structure in the human major histocompatibility complex (MHC), we 
also excluded SNPs in the 26 to 36 megabase region of chromosome six from the data. In analyses both with and without the APOE and MHC regions, LDSC reveals a significant genetic correlation between AD and several of the GIT traits (Fig. 2 and Additional file 1: Table S2).

LDSC reveals a positive and significant genetic correlation $\left(r_{g}\right)$ of $A D$ (without $A P O E$ and $M H C$ regions) with GERD $\left(r_{g}=0.19, P=8.78 \times 10^{-7}\right), P U D\left(r_{g}=0.26, P\right.$ $\left.=2.92 \times 10^{-4}\right), P G M\left(r_{g}=0.15, P=1.43 \times 10^{-4}\right)$, gastritis-duodenitis $\left(r_{g}=0.19, P=5.40 \times 10^{-3}\right)$, IBS $\left(r_{g}=0.16, P=2.36 \times 10^{-2}\right)$, and diverticular disease $\left(r_{g}=0.18\right.$, $\mathrm{P}=1.59 \times 10^{-3}$ ). These results (Fig. 2) are all consistent with findings in SECA. Moreover, our results were based on the unconstrained genetic covariance intercept, hence the significance of these estimates may be conservative given the negligible, or complete absence of, sample overlap between the pairs of traits assessed.

Using LDSC, we did not find a significant genetic correlation between AD and IBD $\left(r_{g}=-0.05, P=3.80 \times 10^{-1}\right)$ [Fig. 2 and Additional file 1: Table S2], a result that is partially consistent with our SECA findings-highlighting how SECA differs (a bidirectional assessment of the relationships) as well as complements LDSC. Additional file 1: Table S2, comprehensively describes the findings of these analyses. We also performed cross-trait LDSC analysis assessing the relationship between each of the GWAS included in this study (Fig. 3 and Additional file 1: Table S2). Notably, there was no evidence for a significant relationship of IBD with any of the other GIT disorders, except IBS $\left(r_{g}=0.14, P=4.41 \times 10^{-3}\right)$ [Fig. 3 and Additional file 1: Table S2]. Conversely, we found a significant genetic correlation between all the other pairs of GIT disorders (Fig. 3 and Additional file 1: Table S2). It is noteworthy that the GWAS for medication use in PUD and GERD (PGM) was strongly correlated with disorders of the gastric mucosa (PUD $\left[r_{g}=0.76, P=4.41 \times 10^{-101}\right]$, gastritis-duodenitis $\left[r_{g}=0.76, P=\right.$ $\left.4.41 \times 10^{-20}\right]$ and GERD $\left.\left[r_{g}=0.99, P=0.000\right]\right)$, supporting its inclusion in the present study (Fig. 3 and Additional file 1: Table S2).

\section{SNPs and loci shared by AD and GIT disorders}

Leveraging the significant genetic overlap and correlation as well as the substantial sample sizes of GERD and PUD, we performed cross-disorder metaanalyses of $A D$ with each of these disorders. PGM has a very large number of cases and overall sample size (Table 1$)$ and is strongly correlated with GERD ( $r_{g}$ $=0.99, P=0.000)$ and PUD $\left(r_{g}=0.76, P=4.41 \times 10^{-101}\right)$ [Fig. 3 and Additional file 1: Table S2], hence, we utilised it in meta-analysis with AD. Our analyses identified shared SNPs and susceptibility loci, some of which are novel for both AD and GIT disorders. The primary objective of this analysis was to identify SNPs and loci which were not genome-wide significant in the individual AD or GIT disorder GWAS (i.e., $5 \times 10^{-8}<\mathrm{P}_{\text {GWAS-data }}<0.05$ ) but reached genome-wide significance $\left(P_{\text {meta-analysis }}<5 \times 10^{-8}\right)$ following a meta-analysis (Table 4). We additionally identified SNPs and loci which were already established (genomewide significant, $\mathrm{P}_{\text {GWAS-data }}<5 \times 10^{-8}$ ) in AD (Sentinel AD SNPs/loci), which were also significantly associated with a GIT disorder, and vice versa, following the GWAS meta-analysis.

\section{$A D$ and PGM GWAS meta-analysis}

A total of 42 SNPs reached genome-wide significance $\left(P_{\text {meta-analysis }}<5 \times 10^{-8}\right)$ in the cross-disorder meta-analysis of AD and PGM GWAS (Additional file 1 : Table S3). None of these 42 SNPs was genome-wide significant in the individual AD or PGM GWAS (before meta-analysis) $\left[P_{\text {GWAS-data }}>5 \times 10^{-8}\right]$ but they were at least nominally significant $\left(P_{\text {GWAS-data }}<0.05\right)$ in each of the traits $\left(5 \times 10^{-8}<P_{\text {GWAS-data }}<0.05\right)$. Of the 42 genome-wide significant SNPs, 11 were independent (at $\left.r^{2}<0.6\right)$, from which we characterised seven lead SNPs at seven genomic loci $\left(r^{2}<0.1\right)$ [Table 4]. That is, seven independent loci reached genome-wide significance for the AD and PGM. A search in the PhenoScanner [45] (accessed on 04/05/2021), revealed that one of the 11 independent SNPs, rs11083749 (on chromosome 19q13.32, NECTIN2), has been reported for association with AD at a genome-wide significant level. Our study provides evidence that this SNP and locus are also associated with PGM given the substantial reduction in the GWAS meta-analysis P-value.

Of the remaining nine independent SNPs, at six genomic loci, none was previously found to be associated with AD, GERD, or PUD at a genome-wide level of significance, suggesting them to be novel SNPs and loci for the analysed traits (Table 4). Moreover, the results for m-value posterior probability and the BE Pvalue indicate that all the identified SNPs and loci, except the 3p21.31 locus (SNPs rs709210 [ HYAL2] and rs7642934 [SEMA3F]), are associated with both AD and PGM. The m-value for each of the remaining SNPs (excluding the 3p21.31 locus) was > 0.90, predicting that they have effects in both GWAS (Table 4). Notably, the 3p21.31 locus (HYAL2 and SEMA3F), was subsequently identified to have effects both in AD and GIT-trait (based on the binary effect P-value results) in the meta-analysis of AD and PUD GWAS (Table 4).

We identified an additional 23 SNPs, at three independent loci $\left(r^{2}<0.1\right)$, that reached genome-wide suggestive association $\left(P_{\text {meta-analysis }}<1 \times 10^{-5}\right)$ in the meta-analysis of AD and PGM (Additional file 1: Table S4). Of these, the rs33998678 SNP (at 16q22.1, IL34) is in high LD ( $\left.r^{2}=0.91\right)$ with one of the genomewide significant SNP loci (rs34644948, at 16q22.1, MTSS2, Table 4) identified in the meta-analysis of AD and PGM. The finding, thus, supports the involvement of the locus (16q22.1, MTSS2) in both AD and GERD or PUD (traits represented by PGM GWAS). Similarly, the rs663576 (at 17q21.32, PHOSPHO1) is moderately correlated $\left(r^{2}=0.41\right)$ with another genome-wide significant SNP (rs2584662 at 17q21.33, PHB, Table 4), identified in the metaanalysis. This locus (17q21.33) was reproduced in the meta-analysis of AD and GERD (SNP rs2584662 near PHB), lending support for its involvement in AD and PUD or GERD. Notably, all the three loci reaching suggestive associations were predicted, using m-value and the BE P-value methods, to have effects in both $A D$ and $P G M$.

\section{$A D$ and GERD GWAS meta-analysis}

A meta-analysis of AD and GERD identified a total of 119 SNPs reaching genome-wide significant association $\left(P_{\text {meta-analysis }}<5 \times 10^{-8}\right.$, Additional file 1 : Table S5), none of which was previously genome-wide significant in the individual AD or GERD GWAS $\left(5 \times 10^{-8}<\mathrm{P}_{\mathrm{GWAS} \text {-data }}<0.05\right)$. From these, we characterised 11 independent SNPs $\left(r^{2}<0.6\right)$ and seven lead SNPs $\left(r^{2}<0.1\right)$ at seven genomic loci (Table 4). The identified loci included those implicated in the meta-analysis 
of $A D$ and $P G M$ at a genome-wide level of significance $\left(P_{\text {meta-analysis }}<5 \times 10^{-8}\right)-1 p 31.3,3 p 21.31,6 p 21.32,17 q 21.33$ and $19 q 13.32-a n d$ at a genome-wide suggestive association level $\left(P_{\text {meta-analysis }}<1 \times 10^{-5}\right)-16 q 22.1$ and $1 \mathrm{q} 32.2$.

Also, we found one (nearby) locus, 17q21.32 (SNP rs8067459, ZNF652, Table 4) reaching genome-wide significance in the AD and GERD meta-analysis. This locus was genome-wide suggestive in the AD vs PGM GWAS meta-analysis (SNP rs663576, 17q21.32, LD between rs663576 and rs8067459 = 0.86 ), providing additional evidence for the locus being shared by AD and the GIT disorders (GERD and PUD). An additional 175 independent SNPs at 121 loci reached a genome-wide suggestive association $\left(P_{\text {meta-analysis }}<1 \times 10^{-5}\right)$, reproducing some of the genome-wide significant loci in the $A D$ and $P G M$ or the $A D$ and $G E R D$ meta-analysis. The loci include 1p31.3 (rs2840677, PDE4B), 1q31.1 (rs10753964, BRINP3) and 1q32.2 (rs4147104, CD46) [Additional file 1: Table S6). Thus, the results support the loci being shared by AD and GERD. Other SNPs and loci reproduced at the suggestive level of association (or in high LD with identified loci) are highlighted in Additional file 1: Table S6.

\section{AD and PUD GWAS meta-analysis}

We identified 22 SNPs reaching genome-wide significance in the meta-analysis of AD and PUD GWAS ( $P_{\text {meta-analysis }}<5 \times 10^{-8}$, Additional file 1 : Table $\left.S 7\right)$. From these, we characterised seven independent SNPs at six genomic loci (Table 4) associated with both AD and PUD. Both the m-value (>0.90) and the BE methods predict that the identified SNPs and loci have effects in AD and PUD (Table 4). Of the loci identified in the AD and PGM meta-analysis, four were replicated in the AD and PUD meta-analysis. Two of the four loci, the 19q13.32 (rs28363848 near BCL3), and the 6p21.32 (rs9270599, HLA-DRA), were replicated at a genome-wide level of significance, while the remaining two-rs709210,3p21.31, $P_{(\mathrm{FE})}=5.24 \times 10^{-3}, H Y A L 2 ;$ and rs6695557, $1 \mathrm{p} 31.3, P_{(\mathrm{FE})}=2.94$ $\times 10^{-4}, P D E 4 B$-were replicated at a nominal level (significant reduction in P-value after AD and PUD meta-analysis, Additional file 1: Table S8). The SNP rs530324, at 8p21.1 (SCARA3, Table 4), identified in the AD and PUD meta-analysis, is in strong LD $\left(r^{2}=0.91\right)$ with another SNP (rs4732732, CLU) which reached a suggestive association for $A D$ and PUD (Additional file 1: Table S9). The finding, thus, provides additional evidence for the involvement of the locus (8p21.1) in both AD and PUD. Additional file 1: Table S9, presents 24 independent SNPs, at 21 genomic loci, reaching genome-wide suggestive association $\left(P_{\text {meta-analysis }}<1 \times 10^{-5}\right)$ for $A D$ and PUD.

\section{Shared genomic regions}

Using a colocalization analysis in GWAS-PW [30], we assessed shared genomic regions between AD and each of PGM and GERD (Additional file 1: Table S10). The results of this analysis confirm that all the loci identified in the meta-analyses (except in chromosome 3 ) are shared by AD and the respective GIT traits (model 4 posterior probability [PPA 4] > 0.9, Additional file 1: Table S10). While the findings also suggest that the causal variants might be different (in some of the loci-PPA $3<0.5$ ), we note that when variants in a locus are in strong LD, which is likely the case here, GWAS-PW is limited in its ability to correctly distinguish model 3 (PPA 3) from model 4 (PPA 4) [30]. Additional shared genomic regions, in chromosomes 1, 6, 16, 17 and 19 having PPA $4>0.90$ were identified for $A D$ and the GIT traits (Additional file 1: Table S10). Also, we identified another locus on chromosome 17, having PPA $3>0.80$, and implicating the SNP rs2526380 (17q22, TSPOAP1) in both AD and GERD. The posterior probability that this SNP is a causal variant under model 3 [30] is high at 0.99 (Additional file 1: Table S10).

\section{Results of causal association analysis between $A D$ and GIT disorders}

We assessed the causal relationship between AD (as the outcome variable) and GERD (as the exposure variable) using the two-sample Mendelian randomisation (MR) method. We found no evidence of a causal relationship between AD and GERD, irrespective of the direction of the analysis (AD or GERD as the outcome or exposure variable) [Table 5]. For sensitivity testing, we implemented five additional models of MR analysis-MR-Egger, weighted median, simple mode, weighted mode and the MR-PRESSO (Mendelian Randomization Pleiotropy RESidual Sum and Outlier)-since a true finding will be consistent across the multiple methods. Results of all these methods agree with those of the Inverse Variance Weighted (IVW) model supporting a lack of evidence for a causal association between AD and GERD (Table 5 and Additional file 1: Table S11). We carried out further MR analysis assessing AD against each of PUD, PGM, IBS, diverticular disease, and IBD. Findings similarly reveal no evidence for a causal relationship between AD and each of the GIT-disorders assessed (Additional file 1: Table S11, and Additional file 2: Supplementary Note 2).

We also used the Latent Causal Variable (LCV) approach [32] to test for a causal relationship between AD and each of the GIT disorders. The results of LCV suggest a partial causal influence of gastritis-duodenitis (genetic causal proportion $[\mathrm{GCP}]=-0.69, \mathrm{P}=0.0026$ ), and lansoprazole $(\mathrm{GCP}=-0.38$, $\mathrm{P}=0.001129$ ) on $A D$, Table 6. The result was in the reverse direction for diverticular disease $(G C P=0.23, P=0.000272)$. Using another set of GWAS (Table 6$)$, we applied LCV methods to test the reproducibility of the partial causal association found. None of the partial causal association results was reproduced.

\section{Gene-based association analysis}

Using a gene-based analysis of the SNPs that overlapped between the AD and PGM GWAS, we identified a total of 18,763 protein-coding genes for each of the traits. Applying a threshold P-value of $2.66 \times 10^{-6}(0.05 / 18763-$ Bonferroni correction for testing 18,763 genes), we identified 64 genome-wide significant $\left(P_{\text {gene }}<2.66 \times 10^{-6}\right)$ genes for AD (Additional file 1: Table S12), 75 for PGM (Additional file 1: Table S13), and 44 for GERD (Additional file 1: Table S14). Using the Fisher's Combined P-value (FCP) method, a total of 44 genome-wide significant $\left(P_{F C P}<2.66 \times 10^{-6}\right)$ genes shared by $A D$ and $P G M$ were identified, 11 of which were not previously significant in the individual AD or PGM GWAS (Additional file 1: Table S15). It is noteworthy that some of the identified AD and PGM shared genes are in chromosomal locations found in our meta-analysis, including 1p31.3 (PDE4B), 3p21.31, (SEMA3F, HYAL2), 6p21.32 (HLA-DRA) and 19q13.32 (several apolipoprotein genes). We replicated a similar pattern of findings using the AD and the GERD GWAS (Additional file 1: Table S16).

\section{Biological pathways and mechanisms shared by AD and GIT disorders}


To identify significantly enriched biological pathways, mechanisms, and processes for AD, GIT disorders (GERD and PGM having the largest sample size), or their comorbidity, we performed pathway-based functional enrichment analyses in the g: Profiler platform [43]. These analyses enable us to functionally interpret genes overlapping between $A D$ and GIT disorders and can provide biological insight from their commonalities. First, we investigated genes overlapping AD and GERD (at $P_{\text {gene }}<0.05$, FCP < 0.02) and identified several biological pathways that were overrepresented (Fig. 4 and Additional file 1: Table S17), implying they have a role in the mechanisms underlying both AD and GERD.

Pathways related to membrane trafficking and metabolism, alteration, lowering or inhibition of lipids were significantly enriched for both traits (Additional file 1: Table S17). These included plasma lipoprotein assembly, remodelling, and clearance $\left(P_{\text {adjusted }}=2.01 \times 10^{-3}\right)$, cholesterol metabolism $\left(P_{\text {adjusted }}=4.99 \times 10^{-}\right.$ ${ }^{2}$ ), plasma lipoprotein assembly $\left(P_{\text {adjusted }}=3.45 \times 10^{-5}\right)$, and triglyceride-rich plasma lipoprotein particle $\left(P_{\text {adjusted }}=5.23 \times 10^{-9}\right)$, among others. Also, lipase inhibitors $\left(P_{\text {adjusted }}=6.08 \times 10^{-3}\right)$ and the statin (3-Hydroxy-3-methylglutaryl-coenzyme A reductase inhibitors) pathway $\left(P_{\text {adjusted }}=3.99 \times 10^{-2}\right)$ were significantly enriched for AD and GERD (Additional file 1: Table S17).

Pathways related to the immune system were also overrepresented for both $A D$ and GERD as evidenced by the identification of immune or autoimmunerelated disorders such as asthma $\left(P_{\text {adjusted }}=3.53 \times 10^{-3}\right)$, systemic lupus erythematosus $\left(P_{\text {adjusted }}=7.88 \times 10^{-3}\right)$, and type I diabetes mellitus $\left(P_{\text {adjusted }}=2.47 \times\right.$ $\left.10^{-2}\right)$. Other immune-related pathways identified include intestinal immune network for IgA production $\left(\mathrm{P}_{\text {adjusted }}=4.07 \times 10^{-2}\right)$, programmed cell death protein $1(\mathrm{PD}-1)$ signalling $\left(\mathrm{P}_{\text {adjusted }}=5.24 \times 10^{-3}\right)$, translocation of ZAP-70 to immunological synapse $\left(\mathrm{P}_{\text {adjusted }}=2.44 \times 10^{-3}\right)$, and interferon-gamma signalling pathways $\left(P_{\text {adjusted }}=2.45 \times 10^{-2}\right)$ [Additional file 1: Table S17].

Following enrichment mapping and auto-annotation, the identified biological pathways were clustered into six themes of biological mechanisms, namely: 'lipoprotein particle clearance,' 'receptor signalling pathway,' 'side membrane vesicle and cell adhesion,' 'peptide antigen binding,' 'intestinal immune network,' and 'interferon-gamma signalling' (Fig. 4). Moreover, a pathway-based analysis using genes that were overlapping between the AD and PGM GWAS (at $P_{\text {gene }}<$ $0.05)$ replicated some of the pathways identified for $A D$ and GERD, including 'plasma lipoprotein assembly, remodelling, and clearance' $\left(P_{\text {adjusted }}=3.01 \times 10^{-4}\right)$, 'peptide antigen binding' $\left(\mathrm{P}_{\text {adjusted }}=2.28 \times 10^{-3}\right)$, and 'triglyceride-rich plasma lipoprotein particle' $\left(\mathrm{P}_{\text {adjusted }}=6.60 \times 10^{-8}\right)[$ Additional file $1:$ Table $S 18$, and Additional file 2: Supplementary Note 3].

\section{Discussion}

We present the first comprehensive assessment of the shared genetics of AD and GIT disorders by analysing large scale GWAS summary data using multiple statistical genetics approaches. We found a significant genetic overlap and correlation between AD and each of GERD, PUD, PGM (medications for PUD or GERD), gastritis-duodenitis, IBS, and diverticular disease. These results support evidence of shared genetic susceptibility between AD and these GIT traits. Also, we identified several independent SNPs, susceptibility loci, genes and biological pathways shared by AD and GERD (and by extension, PUD). These findings not only confirm the results of previous observational studies [5-11] which have suggested a co-occurring association of GIT disorders with the risk of AD but also provide novel insights into the mechanisms underlying the observed associations.

In contrast to the positive genetic correlation between $A D$ and the GIT traits examined, we did not find a significant genetic correlation between $A D$ and IBD using LDSC, which may be due to the relatively small number of cases in the IBD GWAS. Supporting this premise, SECA revealed a significant genetic overlap between the disorders when AD was assessed as dataset 1 against IBD as dataset 2, but not the other way around. The AD GWAS has a much larger case and total sample size and therefore provides a more robust association on which to condition (select independent) SNPs for concordance analysis. Alternatively, IBD may have a different mechanism from the rest of the GIT disorders and is not associated with AD. This position is suggested by the non-significant genetic correlation of IBD with the other GIT traits, except IBS, and supported by findings in a recent GIT GWAS analysis [25]. Given these results, and the highly significant association between $A D$ and IBD reported in a previous observational study [9], future studies need to further investigate the relationship between AD and IBD using more powerful IBD GWAS, as they become available.

Evidence of significant genetic overlap and correlation reflects not only shared genetic aetiologies (biological pleiotropy) but also suggests a possible causal association between AD and the GIT traits (vertical pleiotropy). Using LCV, we detected a partial causal association between AD and each of gastritisduodenitis, lansoprazole, and diverticular disease. However, when we attempted to reproduce findings for gastritis-duodenitis using another GWAS (also for lansoprazole using PGM), this causal association was not evident. The inconclusive LCV findings should be cautiously interpreted, and a reassessment of the results, in future studies, is warranted. Conversely, all MR analyses provided no evidence for a significant causal relationship between AD and the GIT traits, indicating that shared genetics and common biological pathways may best explain the association between AD and these GIT disorders.

We performed GWAS meta-analysis to identify shared SNPs and susceptibility loci, leveraging the larger sample sizes of the PGM, GERD and PUD GWAS, and the significant pleiotropy between $A D$ and each of these GIT traits. Our meta-analysis of AD and PGM GWAS identified seven shared independent loci reaching genome-wide significance for association with both traits. Several of these loci were also identified in meta-analyses of AD with each of GERD and PUD. Results from 'm-value,' binary effect [38], and GWAS-PW [30] methods, overall, robustly support these results. Moreover, many of the loci, including 1p31.3 (PDE4B), 3p21.31 (SEMA3F, HYAL2), 6p21.32 (HLA-DRA), and 19q13.32 (APOE, APOC2, ERCC2, BCL3, and KLC3 genes) were replicated in gene-based association analyses. Following a search in PhenoScanner, six of these loci-1p31.3 (PDE4B), 1q32.2 (CD46), 3p21.31 (SEMA3F, HYAL2), 6p21.32 (HLA-DQA2, HLA-DRA), 16q22.1 (MTSS2), 17q21.33 (PHB)-have not previously been reported, at a genome-wide level of significance, for AD, GERD, or PUD, indicating they are putative novel loci associated with these traits. The remaining locus (19q13.32), harbouring the $A P O E$ gene, has a well-established association with $A D$, and our results suggest it is also involved in these GIT phenotypes. A previous association of APOE genotype with the gut microbiome [46], and IBD [47, 48] may support current findings. 
Notably, the independent SNP rs12058296 (1p31.3), which reached genome-wide significance, mapped to the phosphodiesterase 4B (PDE4B) gene. Inhibition of $P D E 4 B$ (or its subtypes) has shown promise as a treatment for inflammatory diseases [49-52]. Indeed, consistent recent evidence supports the potent antiinflammatory, pro-cognitive, neuro-regenerative, and memory-enhancing properties of PDE4 inhibitors (PDE4B, in particular [53]), making them plausible therapeutic targets for AD [51,52] and GIT disorders [50]. Other identified independent genome-wide significant SNPs and loci mapped to nearby genes including CD46, SEMA3F, HLA-DRA, MTSS2, PHB, and APOE. The CD46 gene is a complement regulator which is bactericidal to Helicobacter (H) pylori [54] and was also recently identified to be associated with $A D$ in a transcriptome analysis [55], making it a plausible candidate in both $A D$ and $G I T$ disorders.

Using pathway-based analyses, we identified biological pathways, mechanisms and processes significantly enriched for AD and digestive phenotypes (GERD, and PUD). Notably, lipid-related, and autoimmune pathways were overrepresented. There is a close link between autoimmunity and lipid abnormalities [56], and our findings highlight abnormal lipid profiles as potential risks for AD and digestive (GERD and PUD) disorders, consistent with findings in previous studies [57-61]. In AD, for example, hypercholesterolemia is believed to increase the permeability of the blood-brain barrier system, facilitating the entry of peripheral cholesterol into the CNS, and resulting in abnormal cholesterol metabolism in the brain [57, 58]. Amyloidogenesis, alteration of the amyloid precursor protein degradation, accumulation of $A b$, and subsequent cognitive impairment have all been linked with elevated cholesterol in the brain [58, 62-64]. A recent study indicates that an increased level of cholesterol in the brain contributes to AD progression through impaired mitochondrial clearance and interference with the ubiquitin-mediated mitophagy process [65]. While the exact roles of lipids in GIT disorders are unclear, $H$. pylori is believed to cause or worsen abnormal serum lipid profiles through chronic inflammatory processes, and eradication of the infection enhances lipid homeostasis [60, 61].

The mechanisms of association between $\mathrm{AD}$ and lipid dysregulation relate to the 'gut-brain axis', alterations in GIT microbiota and the immune system [12, 58]. This observation is consistent with our findings, revealing the likely potential of (or support for) lipid-lowering medications such as lipase inhibitors and statins (identified in our study) for the management of AD and GIT disorders (GERD and PUD, in particular) or their comorbidity. Lipase inhibitors such as orlistat prevent intestinal dietary lipid absorption, thereby decreasing total plasma triglycerides and cholesterol levels [66, 67], making them a preferred pharmacological treatment for obesity [66]. The acknowledged connection between AD, lipid dysregulation, dysbiosis and the 'gut-brain axis' [12, 58], may support the potential utility of lipase inhibitors in AD. Other lipases, including monoacylglycerol, diacylglycerol, and lipoprotein lipases are involved in AD pathology, and can also effectively be inhibited by orlistat [67]. Thus, we hypothesise that lipase inhibitors may be promising in comorbid AD and GIT disorders.

Statins (cholesterol-lowering medications) are also therapeutically beneficial in AD and GIT disorders [68-72]. Evidence indicates that statins possess antiinflammatory, immune-modulating and gastroprotective properties [68,69], and their active use was associated with a significant PUD risk reduction [68], and H. pylori eradication [70]. Further, statins improve cognitive ability and reduce neurodegeneration risks, making them potentially beneficial in AD [71, 72]. However, there is (controversial) evidence suggesting a paradoxical predisposition to reversible dementia for statins [71, 72]. While this finding has been challenged [71], it highlights a clear need to identify AD patients for whom statins will be beneficial, consistent with the model of personalised health. Hence, we hypothesise that statins may be beneficial in individuals with comorbid AD and GIT disorders.

Our findings have implications for practice and further studies. First, results highlighting lipid-related mechanisms support the roles of abnormal lipid profiles in the aetiologies of the disorders, which may be potential biomarkers for AD and GIT disorders (or their comorbidity). Further investigation of these results in the traits in question is warranted in future experimental studies. Second, our findings underscore the importance of lipid homeostasis. The dietary approach is one effective preventive as well as non-pharmacologic approach for the management of hyperlipidaemia, and overall, this is consistent with findings in this study. Indeed, adherence to a 'Mediterranean' diet (low in lipids) is recognised as beneficial both in AD [73] and GIT disorders [74]. Thus, a recommendation for healthy diets, early in life, may form part of the lifestyle modifications for preventing AD and GIT disorders. Again, the clinical usefulness and relevance of this recommendation will need to be further investigated or validated. Third, our study identifies lipase inhibitors and statin pathways in the mechanisms of $A D$ and GIT disorders, which may be a potential therapeutic avenue to explore in the disorders. Hence, we hypothesise that individuals with comorbid AD and GIT traits may gain benefits from these therapies. There is a need to test this hypothesis using appropriate study designs including randomised control trials. Fourth, our study implicates the $P D E 4 B$, and given the evidence in the literature [50-53], we propose that treatment targeted at its inhibition may be promising in comorbid AD and GIT traits. Future studies, including randomised control trials, are needed to test these hypotheses. Lastly, while we note that our findings do not necessarily indicate that AD and GIT disorders will always co-occur, the finding of significant genetic overlap and correlation between them support their shared biology. Thus, it may be beneficial for healthcare providers to probe signs or symptoms of impaired cognition in individuals presenting with GIT disorders and vice versa, to improve possible early detection.

The use of multiple, complementary statistical genetic approaches enables a comprehensive analysis of the genetic associations between AD and GIT disorders and is a major strength of this study. Also, we analysed well-powered GWAS data, meaning our findings are generally not affected by the small sample sizes, possible reverse causality, or confounders that conventional observational studies often suffer from. Importantly, biases due to potential sample overlap do not apply in the present study. First, the results of the genetic covariance intercept in LDSC analysis indicates the absence of sample overlap for most pairs of $A D$ and GIT traits assessed and negligible chances of such occurrence between AD and GERD (genetic covariance intercept $=0.0133$, se = 0.005) as well as between AD and PGM (genetic covariance intercept $=0.0135$, se $=0.006$ ). Second, we obtained a consistent result across several methods, many of which are not affected by, or can adjust for, sample overlap-LDSC (unconstrained intercept), LCV and RE2C (GWAS meta-analysis).

Nonetheless, our study has limitations that should be considered alongside the present findings. First, the GWAS for AD combined clinically diagnosed cases of $A D$ with proxies (AD-by-proxy-individuals whose parents were diagnosed with AD). Given the high correlation between the GWAS with and without the 'ADby-proxy' cases [24], we argue as did others [24] that combining them is valid, especially for sample size improvement, which is critical to ensuring adequately powered GWAS analysis. Second, there are suggested limitations around false positives in MR analysis due to possible violation of some of its underlying assumptions. In the present study, we used multiple MR models to complement the respective strengths and weaknesses of the methods. Also, we tested for horizontal pleiotropy using the MR-Egger intercept, and to exclude pleiotropic SNPs (where present), we applied MR-PRESSO. Importantly, we found no

Page 9/21 
evidence for a significant causal association in the present study ruling out the possibility of false-positive results. Third, analyses were restricted to participants of mainly European ancestry in our study, thus, findings may not be generalisable to other ancestries. Fourth, GIT phenotypes GWAS were combinations of several data sources: primary care, hospital admission, medication use, and self-reported records. While there is a potential for misdiagnosis or accuracy of self-reported data, their use is well justified given the correlation in effect sizes of the data with other sources [25]. Moreover, additional data from other sources including ICD-10 were utilised with consistent results across these GWAS.

\section{Conclusions}

In conclusion, this study provides novel insights into the long-standing debate and the observed relationship of AD with GIT disorders, implicating shared genetic susceptibility. We found a significant risk increasing (but non-causal) genetic association between AD and each of GERD, PUD, PGM (medications for PUD or GERD), gastritis-duodenitis, IBS, and diverticular disease. Also, we identified independent regions in the genome and genes shared by AD and GIT disorders which may be potential targets for further investigation in the mechanisms of the disorders. Functional enrichment analysis implicates lipids, cholesterol, lipid metabolites and autoimmune-related pathways in the mechanisms of AD, GIT disorders, and potentially, their comorbidity. Notably, our study suggests the potential relevance of statins and lipase inhibitors in AD, GIT disorders or their comorbidity. To our knowledge, this is the first comprehensive study to assess these relationships using statistical genetics approaches. Overall, these findings advance our understanding of the genetic architecture of $A D$, GIT disorders, and their observed co-occurring relationship.

\section{Abbreviations}

AD: Alzheimer's disease

AOR: Adjusted odds ratio

Ab: amyloid-beta

BE: Binary Effects

Cl: Confidence interval

CNS: Central nervous system

CTG: Genetics of Complex Traits

FCP: Fisher Combined P-value

FE: Fixed Effects

FUMA: Functional Mapping and Annotation

GCP: Genetic causality proportion

GERD: Gastroesophageal reflux disease

GIT: Gastrointestinal tract

GWAS: Genome-wide association studies

GWAS-PW: Pairwise GWAS

H. pylori: Helicobacter pylori

HR: Hazard ratio

IBD: Inflammatory bowel disease

IBS: Irritable bowel syndrome

IVs: Instrumental variables

IVW: Inverse variance weighted

LCV: Latent Causal Variable

LD: Linkage disequilibrium

LDSC: Linkage disequilibrium score regression

MAGMA: Generalized gene-set analysis of GWAS data 
MHC: Major histocompatibility complex

MR: Mendelian randomisation

MR-PRESSO: Mendelian randomisation pleiotropy residual sum and outlier

m-value: Posterior probability

$\mathrm{N}$ : Sample size

OR: Odds ratio

P: P-value

P1: P-value for dataset 1

P2: P-value for dataset 2

$\mathrm{P}_{\text {adjusted }}$ : Adjusted P-value

PD-1: programmed cell death protein 1

$\mathrm{P}_{\text {gene: }}$ Gene-based P-value

PGM: Medications for GERD or PUD

PPA: Posterior probability of association

$\mathrm{P}_{\text {permuted: }}$ Permuted P-value

PPI: Proton-pump inhibitors

PUD: Peptic ulcer disease

RCTs: randomised control trials

RE2: Modified Random Effect

RE2C: Modified random-effects correlation

$\mathrm{r}_{\mathrm{g}}$ : genetic correlation

SECA: SNP effect concordance analysis

SNP: Single nucleotide polymorphism

UK: United Kingdom

UKB: United Kingdom Biobank

US: United States

\section{Declarations}

\section{Ethics approval and consent to participate}

This study is a secondary analysis of existing GWAS summary data from public repositories. Specific and relevant ethics approval for each of the data utilised is presented in the associated publications as described in the subsection for GWAS summary data. No additional ethics approval is required for the conduct of the present study.

\section{Consent for publication}

Not applicable

\section{Availability of data and materials}

All data generated during this study are included in the published article and its supplementary section. GWAS summary statistics data analysed were sourced from international research consortia and public repositories as described in the subsection for GWAS summary data. The data are freely available and accessible online through the links and references provided within this study. 


\section{Competing interests}

The authors declare that they have no competing interests

\section{Funding}

This research was supported through funding to SML from the National Health and Medical Research Council (Australia; APP1161706, APP1191535). Funders have no influence on the design, analysis or interpretation of findings in this study.

\section{Authors' contributions}

Conceived the Study: EOA., TP., SML; designed the study: EOA., EKO., TP., SML.; conducted the analysis: EOA; interpreted the results: EOA., EKO., DRN., TP., SML.; drafted the manuscript: EOA.; made critical revisions to the manuscript: EOA., EKO., DRN., TP., SML; Funding: SML All authors read and approved the final manuscript.

\section{Acknowledgements}

The GWAS data analysed in the present study were sourced from several databases and international research groups/consortia. We express our appreciation to all these research groups, consortia and the UKB for providing us access to their data.

\section{References}

1. Prince MJ. World Alzheimer Report 2015: the global impact of dementia: an analysis of prevalence, incidence, cost and trends: Alzheimer's Disease International; 2015.

2. Cummings J, Lee G, Ritter A, Sabbagh M, Zhong K. Alzheimer's disease drug development pipeline: 2019. Alzheimer's \& Dementia: Translational Research \& Clinical Interventions. 2019;5:272-93.

3. Takizawa C, Thompson PL, van Walsem A, Faure C, Maier WC. Epidemiological and economic burden of Alzheimer's disease: a systematic literature review of data across Europe and the United States of America. J Alzheimers Dis. 2015;43(4):1271-84.

4. Levey Al. Progress with Treatments for Alzheimer's Disease. New England Journal of Medicine. 2021;384(18):1762-3.

5. Momtaz YA, Hamid TA, Ibrahim R. Gastritis May Boost Odds of Dementia. American Journal of Alzheimer's Disease \& Other Dementiasr. 2014;29(5):4526.

6. Gomm W, von Holt K, Thomé F, Broich K, Maier W, Fink A, et al. Association of proton pump inhibitors with risk of dementia: a pharmacoepidemiological claims data analysis. JAMA neurology. 2016;73(4):410-6.

7. Badiola N, Alcalde V, Pujol A, Münter L-M, Multhaup G, Lleo A, et al. The proton-pump inhibitor lansoprazole enhances amyloid beta production. PloS one. 2013;8(3):e58837.

8. Jiang C, Li G, Huang P, Liu Z, Zhao B. The gut microbiota and Alzheimer's disease. Journal of Alzheimer's Disease. 2017;58(1):1-15.

9. Zhang B, Wang HE, Bai Y-M, Tsai S-J, Su T-P, Chen T-J, et al. Inflammatory bowel disease is associated with higher dementia risk: a nationwide longitudinal study. Gut. 2021;70(1):85-91.

10. Chen C-H, Lin C-L, Kao C-H. Irritable Bowel Syndrome Is Associated with an Increased Risk of Dementia: A Nationwide Population-Based Study. PLOS ONE. 2016;11(1):e0144589.

11. Doulberis M, Kotronis G, Gialamprinou D, Polyzos SA, Papaefthymiou A, Katsinelos P, et al. Alzheimer's disease and gastrointestinal microbiota; impact of Helicobacter pylori infection involvement. Int J Neurosci. 2021;131(3):289-301.

12. Guo T, Zhang D, Zeng Y, Huang TY, Xu H, Zhao Y. Molecular and cellular mechanisms underlying the pathogenesis of Alzheimer's disease. Molecular Neurodegeneration. 2020;15(1):40.

13. Selkoe DJ, Hardy J. The amyloid hypothesis of Alzheimer's disease at 25 years. EMBO molecular medicine. 2016;8(6):595-608.

14. Kim SY, Min C, Oh DJ, Choi HG. Reciprocal association between depression and peptic ulcers: Two longitudinal follow-up studies using a national sample cohort. Scientific Reports. 2020;10(1):1749.

15. Adewuyi EO, Mehta D, Sapkota Y, Sapkota Y, Yoshihara K, Nyegaard M, et al. Genetic analysis of endometriosis and depression identifies shared loci and implicates causal links with gastric mucosa abnormality. Human Genetics. 2021;140(3):529-52.

16. Menozzi E, Macnaughtan J, Schapira AHV. The gut-brain axis and Parkinson disease: clinical and pathogenetic relevance. Annals of Medicine. 2021;53(1):611-25.

17. 17.

18. Pellegrini C, Antonioli L, Colucci R, Blandizzi C, Fornai M. Interplay among gut microbiota, intestinal mucosal barrier and enteric neuro-immune system: a common path to neurodegenerative diseases? Acta Neuropathologica. 2018;136(3):345-61.

19. Gries M, Christmann A, Schulte S, Weyland M, Rommel S, Martin M, et al. Parkinson mice show functional and molecular changes in the gut long before motoric disease onset. Molecular Neurodegeneration. 2021;16(1):34.

20. Sun Y, Sommerville NR, Liu JYH, Ngan MP, Poon D, Ponomarev ED, et al. Intra-gastrointestinal amyloid- $\beta 1$ - 42 oligomers perturb enteric function and induce Alzheimer's disease pathology. The Journal of physiology. 2020;598(19):4209-23. 
21. Khan MA, Yuan Y, Iqbal U, Kamal S, Khan M, Khan Z, et al. No Association Linking Short-Term Proton Pump Inhibitor Use to Dementia: Systematic Review and Meta-analysis of Observational Studies. Official journal of the American College of Gastroenterology | ACG. 2020;115(5):671-8.

22. Zhang Y, Liang M, Sun C, Song EJ, Cheng C, Shi T, et al. Proton pump inhibitors use and dementia risk: a meta-analysis of cohort studies. European Journal of Clinical Pharmacology. 2020;76(2):139-47.

23. Wu C-L, Lei W-Y, Wang J-S, Lin C-E, Chen C-L, Wen S-H. Acid suppressants use and the risk of dementia: A population-based propensity score-matched cohort study. PLOS ONE. 2020;15(11):e0242975.

24. Jansen IE, Savage JE, Watanabe K, Bryois J, Williams DM, Steinberg S, et al. Genome-wide meta-analysis identifies new loci and functional pathways influencing Alzheimer's disease risk. Nature genetics. 2019;51(3):404-13.

25. Wu Y, Murray GK, Byrne EM, Sidorenko J, Visscher PM, Wray NR. GWAS of peptic ulcer disease implicates Helicobacter pylori infection, other gastrointestinal disorders and depression. Nature Communications. 2021;12(1):1146.

26. An J, Gharahkhani P, Law MH, Ong J-S, Han X, Olsen CM, et al. Gastroesophageal reflux GWAS identifies risk loci that also associate with subsequent severe esophageal diseases. Nature Communications. 2019;10(1):4219.

27. Watanabe K, Stringer S, Frei O, Umićević Mirkov M, de Leeuw C, Polderman TJC, et al. A global overview of pleiotropy and genetic architecture in complex traits. Nat Genet. 2019;51(9):1339-48.

28. Nyholt DR. SECA: SNP effect concordance analysis using genome-wide association summary results. Bioinformatics. 2014;30(14):2086-8.

29. Bulik-Sullivan BK, Loh P-R, Finucane HK, Ripke S, Yang J, Patterson N, et al. LD Score regression distinguishes confounding from polygenicity in genomewide association studies. Nature Genetics. 2015;47(3):291-5.

30. Pickrell JK, Berisa T, Liu JZ, Ségurel L, Tung JY, Hinds DA. Detection and interpretation of shared genetic influences on 42 human traits. Nature genetics. 2016;48(7):709.

31. Sekula P, Del Greco M F, Pattaro C, Köttgen A. Mendelian Randomization as an Approach to Assess Causality Using Observational Data. J Am Soc Nephrol. 2016;27(11):3253-65.

32. O'Connor LJ, Price AL. Distinguishing genetic correlation from causation across 52 diseases and complex traits. Nature Genetics. 2018;50(12):1728-34.

33. Purcell S, Neale B, Todd-Brown K, Thomas L, Ferreira MA, Bender D, et al. PLINK: a tool set for whole-genome association and population-based linkage analyses. The American journal of human genetics. 2007;81(3):559-75.

34. Han B, Eskin E. Random-effects model aimed at discovering associations in meta-analysis of genome-wide association studies. The American Journal of Human Genetics. 2011;88(5):586-98.

35. Lee $\mathrm{CH}$, Eskin E, Han B. Increasing the power of meta-analysis of genome-wide association studies to detect heterogeneous effects. Bioinformatics. 2017;33(14):i379-i88.

36. Watanabe K, Taskesen E, van Bochoven A, Posthuma D. Functional mapping and annotation of genetic associations with FUMA. Nature Communications. 2017;8(1):1826.

37. Adewuyi EO, Sapkota Y, Consortium IE, Team aR, Consortium IHG, Auta A, et al. Shared Molecular Genetic Mechanisms Underlie Endometriosis and Migraine Comorbidity. Genes. 2020;11(3):268.

38. Han B, Eskin E. Interpreting Meta-Analyses of Genome-Wide Association Studies. PLOS Genetics. 2012;8(3):e1002555.

39. Bowden J, Davey Smith G, Haycock PC, Burgess S. Consistent estimation in Mendelian randomization with some invalid instruments using a weighted median estimator. Genetic epidemiology. 2016;40(4):304-14.

40. Bowden J, Davey Smith G, Burgess S. Mendelian randomization with invalid instruments: effect estimation and bias detection through Egger regression. International journal of epidemiology. 2015;44(2):512-25.

41. Verbanck M, Chen C-y, Neale B, Do R. Detection of widespread horizontal pleiotropy in causal relationships inferred from Mendelian randomization between complex traits and diseases. Nature genetics. 2018;50(5):693-8.

42. Cuéllar-Partida G, Lundberg M, Kho PF, D’Urso S, Gutiérrez-Mondragón LF, Ngo TT, et al. Complex-Traits Genetics Virtual Lab: A community-driven web platform for post-GWAS analyses. bioRxiv. 2019:518027.

43. Raudvere U, Kolberg L, Kuzmin I, Arak T, Adler P, Peterson H, et al. g:Profiler: a web server for functional enrichment analysis and conversions of gene lists (2019 update). Nucleic Acids Research. 2019;47(W1):W191-W8.

44. Reimand J, Isserlin R, Voisin V, Kucera M, Tannus-Lopes C, Rostamianfar A, et al. Pathway enrichment analysis and visualization of omics data using g:Profiler, GSEA, Cytoscape and EnrichmentMap. Nature Protocols. 2019;14(2):482-517.

45. Staley JR, Blackshaw J, Kamat MA, Ellis S, Surendran P, Sun BB, et al. PhenoScanner: a database of human genotype-phenotype associations. Bioinformatics. 2016;32(20):3207-9.

46. Parikh IJ, Estus JL, Zajac DJ, Malik M, Maldonado Weng J, Tai LM, et al. Murine Gut Microbiome Association With APOE Alleles. Frontiers in Immunology. 2020;11(200).

47. Al-Meghaiseeb ES, Al-Otaibi MM, Al-Robayan A, Al-Amro R, Al-Malki AS, Arfin M, et al. Genetic association of apolipoprotein E polymorphisms with inflammatory bowel disease. World journal of gastroenterology: WJG. 2015;21(3):897.

48. Glapa-Nowak A, Szczepanik M, Iwańczak B, Kwiecień J, Szaflarska-Popławska AB, Grzybowska-Chlebowczyk U, et al. Apolipoprotein E variants correlate with the clinical presentation of paediatric inflammatory bowel disease: A cross-sectional study. World J Gastroenterol. 2021;27(14):1483-96.

49. Li H, Zuo J, Tang W. Phosphodiesterase-4 Inhibitors for the Treatment of Inflammatory Diseases. Front Pharmacol. 2018;9:1048-. 
50. Spadaccini M, D'Alessio S, Peyrin-Biroulet L, Danese S. PDE4 Inhibition and Inflammatory Bowel Disease: A Novel Therapeutic Avenue. Int J Mol Sci. 2017;18(6):1276.

51. Richter W, Menniti FS, Zhang H-T, Conti M. PDE4 as a target for cognition enhancement. Expert Opin Ther Targets. 2013;17(9):1011-27.

52. Sanders O, Rajagopal L. Phosphodiesterase Inhibitors for Alzheimer's Disease: A Systematic Review of Clinical Trials and Epidemiology with a Mechanistic Rationale. J Alzheimers Dis Rep. 2020;4(1):185-215.

53. Tibbo AJ, Baillie GS. Phosphodiesterase 4B: Master Regulator of Brain Signaling. Cells. 2020;9(5):1254.

54. Basmarke-Wehelie R, Sjölinder H, Jurkowski W, Elofsson A, Arnqvist A, Engstrand L, et al. The complement regulator CD46 is bactericidal to Helicobacter pylori and blocks urease activity. Gastroenterology. 2011;141(3):918-28.

55. Nho K, Nudelman K, Allen M, Hodges A, Kim S, Risacher SL, et al. Genome-wide transcriptome analysis identifies novel dysregulated genes implicated in Alzheimer's pathology. Alzheimer's \& Dementia. 2020;16(9):1213-23.

56. Bagchi S, Genardi S, Wang C-R. Linking CD1-Restricted T Cells With Autoimmunity and Dyslipidemia: Lipid Levels Matter. Frontiers in Immunology. 2018;9(1616).

57. Chen H, Du Y, Liu S, Ge B, Ji Y, Huang G. Association between serum cholesterol levels and Alzheimer's disease in China: a case-control study. International Journal of Food Sciences and Nutrition. 2019;70(4):405-11.

58. Kao Y-C, Ho P-C, Tu Y-K, Jou I-M, Tsai K-J. Lipids and Alzheimer's Disease. International Journal of Molecular Sciences. $2020 ; 21(4): 1505$.

59. Kao Y-C, Ho P-C, Tu Y-K, Jou I, Tsai K-J. Lipids and Alzheimer's disease. Int J Mol Sci. 2020;21(4):1505.

60. Lamb A, Chen L-F. Role of the Helicobacter pylori-induced inflammatory response in the development of gastric cancer. J Cell Biochem. 2013;114(3):4917.

61. Iwai N, Okuda T, Oka K, Hara T, Inada Y, Tsuji T, et al. Helicobacter pylori eradication increases the serum high density lipoprotein cholesterol level in the infected patients with chronic gastritis: A single-center observational study. PLOS ONE. 2019;14(8):e0221349.

62. Di Paolo G, Kim T-W. Linking lipids to Alzheimer's disease: cholesterol and beyond. Nature Reviews Neuroscience. 2011;12(5):284-96.

63. Kojro E, Gimpl G, Lammich S, März W, Fahrenholz F. Low cholesterol stimulates the nonamyloidogenic pathway by its effect on the a-secretase ADAM 10. Proceedings of the national academy of sciences. 2001;98(10):5815-20.

64. Vance JE, Hayashi H, Karten B. Cholesterol homeostasis in neurons and glial cells. Seminars in Cell \& Developmental Biology. 2005;16(2):193-212.

65. Roca-Agujetas V, Barbero-Camps E, de Dios C, Podlesniy P, Abadin X, Morales A, et al. Cholesterol alters mitophagy by impairing optineurin recruitment and lysosomal clearance in Alzheimer's disease. Molecular Neurodegeneration. 2021;16(1):15.

66. Henness S, Perry CM. Orlistat. Drugs. 2006;66(12):1625-56.

67. Du J, Wang Z. Therapeutic potential of lipase inhibitor orlistat in Alzheimer's disease. Medical Hypotheses. 2009;73(5):662-3.

68. Lin C-J, Liao W-C, Chen Y-A, Lin H-J, Feng C-L, Lin C-L, et al. Statin Therapy Is Associated with Reduced Risk of Peptic Ulcer Disease in the Taiwanese Population. Front Pharmacol. 2017;8:210-

69. Pantea M, Negovan A, Voidăzan S, Macarie M, Mocan S, Băţagă S. Statins and gastroduodenal endoscopic lesions: A case-control study. Medicine (Baltimore). 2018;97(50):e13579-e.

70. Nseir W, Diab H, Mahamid M, Abu-Elheja O, Samara M, Abid A, et al. Randomised clinical trial: simvastatin as adjuvant therapy improves significantly the Helicobacter pylori eradication rate-a placebo-controlled study. Aliment Pharmacol Ther. 2012;36(3):231-8.

71. Schultz BG, Patten DK, Berlau DJ. The role of statins in both cognitive impairment and protection against dementia: a tale of two mechanisms. Transl Neurodegener. 2018;7:5-

72. Torrandell-Haro G, Branigan GL, Vitali F, Geifman N, Zissimopoulos JM, Brinton RD. Statin therapy and risk of Alzheimer's and age-related neurodegenerative diseases. Alzheimer's \& Dementia: Translational Research \& Clinical Interventions. 2020;6(1):e12108.

73. van den Brink AC, Brouwer-Brolsma EM, Berendsen AAM, van de Rest O. The Mediterranean, Dietary Approaches to Stop Hypertension (DASH), and Mediterranean-DASH Intervention for Neurodegenerative Delay (MIND) Diets Are Associated with Less Cognitive Decline and a Lower Risk of Alzheimer's Disease-A Review. Advances in Nutrition. 2019;10(6):1040-65.

74. Elmaliklis I-N, Liveri A, Ntelis B, Paraskeva K, Goulis I, Koutelidakis AE. Increased Functional Foods' Consumption and Mediterranean Diet Adherence May Have a Protective Effect in the Appearance of Gastrointestinal Diseases: A Caseâ»Control Study. Medicines (Basel). 2019;6(2):50.

\section{Tables}

Table 1: Summary of GWAS datasets analysed 


\begin{tabular}{|c|c|c|c|c|c|c|}
\hline $\mathrm{S} / \mathrm{N}$ & GWAS & Cases & Control & $\begin{array}{l}\text { Sample } \\
\text { size }\end{array}$ & Ancestry & Phenotype source/definition \\
\hline 1 & Alzheimer's disease (AD) & 71,880 & 383,378 & 455,258 & \multirow[t]{10}{*}{ European } & Clinically diagnosed and UKB AD-by-proxy [23] \\
\hline 2 & Peptic ulcer disease (PUD) & 16,666 & 439,661 & 456,327 & & \multirow{5}{*}{$\begin{array}{l}\text { UKB data code for case definition was from death register, primary } \\
\text { care, hospital admissions data, self-report only, and other sources as } \\
\text { described in the corresponding publication [24] }\end{array}$} \\
\hline 3 & $\begin{array}{l}\text { Irritable Bowel Syndrome } \\
\text { (IBS) }\end{array}$ & 28,518 & 426,803 & 455,321 & & \\
\hline 4 & $\begin{array}{l}\text { Inflammatory Bowel } \\
\text { Disease (IBD) }\end{array}$ & 7045 & 426803 & 456,327 & & \\
\hline 5 & $\begin{array}{l}\text { PUD and GERD medication } \\
(\mathrm{PGM})^{\star}\end{array}$ & 90,175 & 366,152 & 456,327 & & \\
\hline 6 & Diverticular Disease & 14,028 & 286,763 & 300,791 & & \\
\hline 7 & $\begin{array}{l}\text { Gastroesophageal Reflux } \\
\text { Disease (GERD) }\end{array}$ & 71,522 & 261,079 & 332,601 & & Combined datasets from the UKB and the QSKIN study [25] \\
\hline 8 & $\begin{array}{l}\text { Gastritis-Duodenitis- } \\
\text { UKBB_SAIGE_PheCode_535 }\end{array}$ & 28,941 & 378,124 & 407,065 & & \multirow[t]{2}{*}{$\begin{array}{l}\text { Full European subset of the phenotype definition in the UKB from the } \\
\text { Lee Lab from (https://www.leelabsg.org/resources) }\end{array}$} \\
\hline 9 & $\begin{array}{l}\text { Diverticulosis and } \\
\text { diverticulitis- } \\
\text { UKBB_PheCode_562 }\end{array}$ & 27,311 & 334,783 & 362,094 & & \\
\hline 10. & Gastritis-Duodenitis & & & & & UKB ICD10, data field K29 \\
\hline
\end{tabular}

AD: Alzheimer's disease, GERD: gastroesophageal reflux disease, PUD: peptic ulcer disease, IBS: irritable bowel disease, IBD: inflammatory bowel disease. UKB: United Kingdom Biobank. The 'clinically diagnosed AD' combined data from three case-control cohorts $(N=79,145)$. 'AD-by proxy' data were based on the UKB phenotype definition of individuals whose biological parents were affected by AD. The parent's current age, and where relevant, age at death were reported along with this UKB GWAS data. The genetic correlation between the 'clinically diagnosed AD' and the 'AD-by proxy' is high at 0.81 [23], providing strong evidence or justification for combining them as more comprehensively described in the associated publication [23]. *PGM: medications for PUD and GERD. Note: Supplementary Table 1 describes these GWAS, and others used for replication studies more comprehensively, providing links to their respective sources.

Table 2: Results of genetic overlap assessment between AD (P1) and GERD (P2)

\begin{tabular}{|lllllll|}
\hline P1 & P2 & Ind-SNPs-overlapping & Concordant & Prop-Overlap & OR & $\begin{array}{l}\text { P } \\
\text { Fishers-exact }\end{array}$ \\
\hline$\leq 1$ & $\leq 1$ & 26963 & 13955 & 0.52 & 1.18 & $4.65 \times 10^{-11}$ \\
\hline 0.9 & 0.9 & 24237 & 12653 & 0.52 & 1.19 & $7.46 \times 10^{-12}$ \\
\hline 0.8 & 0.8 & 21551 & 11289 & 0.52 & 1.21 & $3.02 \times 10^{-12}$ \\
\hline 0.7 & 0.7 & 18759 & 9860 & 0.53 & 1.23 & $2.64 \times 10^{-12}$ \\
\hline 0.6 & 0.6 & 16001 & 8483 & 0.53 & 1.27 & $2.48 \times 10^{-14}$ \\
\hline 0.5 & 0.5 & 13297 & 7081 & 0.53 & 1.30 & $7.54 \times 10^{-14}$ \\
\hline 0.4 & 0.4 & 10432 & 5603 & 0.54 & 1.35 & $3.42 \times 10^{-14}$ \\
\hline 0.3 & 0.3 & 7638 & 4173 & 0.55 & 1.45 & $6.22 \times 10^{-16}$ \\
\hline 0.2 & 0.2 & 4936 & 2755 & 0.56 & 1.6 & $2.86 \times 10^{-16}$ \\
\hline 0.1 & 0.1 & 2283 & 1315 & 0.58 & 1.85 & $4.06 \times 10^{-13}$ \\
\hline 0.05 & 0.05 & 1015 & 585 & 0.58 & 1.84 & $1.74 \times 10^{-6}$ \\
\hline 0.05 & 0.01 & 322 & 199 & 0.62 & 2.59 & $3.66 \times 10^{-5}$ \\
\hline 0.01 & 0.05 & 439 & 266 & 0.61 & 2.39 & $1.03 \times 10^{-5}$ \\
\hline 0.01 & 0.01 & 152 & 93 & 0.61 & 2.34 & $1.47 \times 10^{-2}$ \\
\hline
\end{tabular}

AD: Alzheimer's disease, GERD: gastroesophageal reflux disease, $P 1$ : $P$-value for the dataset, $P 2$ : $P$-value for dataset 2, P1P2snp: Independent SNPs overlapping AD (P1) and GERD (P2) at each of the SNP subsets, Concord: number of concordant SNPs, SNP: Single Nucleotide Polymorphism, Ftest: Fisher's Exact test, OR: Odds ratio for the effect direction concordance association test for P1 and P2. Pval: Fisher's exact P-value for the effect direction concordance association test between $A D(P 1)$ and GERD (P2).

Table 3: Summarised SECA results: genetic overlap between AD and GIT disorders 


\begin{tabular}{|c|c|c|c|}
\hline Test in SECA & $\begin{array}{l}\text { SNP subsets ratio } \\
(144 \text { total })(O R>1, P<0.05)\end{array}$ & $P_{\text {permuted }}$ & Interpretation \\
\hline $\mathrm{AD}(\mathrm{P} 1)$ vs GERD (P2) & 144 & 0.000999 & Significant \\
\hline GERD (P1) vs AD (P2) & 144 & 0.000999 & Significant \\
\hline AD (P1) vs Gastritis-D (P2) & 144 & 0.000999 & Significant \\
\hline Gastritis-D (P1) vs AD (P2) & 135 & 0.000999 & Significant \\
\hline$A D(P 1)$ vs PGM (P2) & 144 & 0.000999 & Significant \\
\hline PGM (P1) vs AD (P2) & 141 & 0.000999 & Significant \\
\hline$A D(P 1)$ vs PUD (P2) & 137 & 0.000999 & Significant \\
\hline PUD (P1) vs AD & 133 & 0.000999 & Significant \\
\hline$A D(P 1)$ vs IBS (P2) & 134 & 0.000999 & Significant \\
\hline IBS (P1) vs AD (P2) & 118 & 0.000999 & Significant \\
\hline$A D(P 1)$ vs Diverticular Disease & 111 & 0.000999 & Significant \\
\hline Diverticular Disease (P1) vs AD (P2) & 97 & 0.002 & Significant \\
\hline$A D(P 1)$ vs IBD ${ }^{a}(P 2)$ & 42 & 0.025 & Significant \\
\hline $\mathrm{IBD}^{\mathrm{a}}(\mathrm{P} 2)$ vs $\mathrm{AD}(\mathrm{P} 2)$ & 9 & 0.122 & Not significant \\
\hline$A D(P 1)$ vs Inflammatory Bowel Disease ${ }^{b}(P 2)$ & 34 & 0.041 & Significant \\
\hline Inflammatory Bowel Disease ${ }^{\mathrm{b}}(\mathrm{P} 1)$ vs $\mathrm{AD}(\mathrm{P} 2)$ & 22 & 0.0599 & Not significant \\
\hline
\end{tabular}

AD: Alzheimer's disease, GIT: gastro-intestinal tract, GERD: gastroesophageal reflux disease, PUD: peptic ulcer disease, PGM: medications for GERD and PUD, IBS: irritable bowel syndrome, IBD: Inflammatory bowel disease. P1: P-value for the dataset, P2: P-value for dataset 2, SNP: Single Nucleotide Polymorphism, OR: Odds ratio for the effect direction concordance association test for P1 and P2. Note: we used two different IBD GWAS having different samples (cases) to assess the relationship between AD and IBD. The first IBD ${ }^{a}$ GWAS was sourced from WU et al., 2021 [2] while the second IBD ${ }^{b}$ GWAS was sourced from the GWAS atlas (ftp://ftp.sanger.ac.uk/pub/consortia/ibdgenetics/iibdgc-trans-ancestry-filtered-summary-stats.tgz) [6]. The results suggest greater sample size for cases of IBD produced improved overlap with AD, thus indicating that using more powerful GWAS for IBD will likely produce greater genetic overlap with AD.

Table 4: Genome-wide significant independent SNPs and loci for AD and GIT disorders 


\begin{tabular}{|c|c|c|c|c|c|c|c|c|c|c|c|c|}
\hline \multirow{2}{*}{$\begin{array}{l}\text { Independent } \\
\text { SNPS }\end{array}$} & \multirow[t]{2}{*}{ Locus } & \multirow[t]{2}{*}{ Lead SNPs } & \multirow[t]{2}{*}{ Chr } & \multirow[t]{2}{*}{ BP } & \multirow[t]{2}{*}{ EA } & \multirow[t]{2}{*}{ NEA } & \multirow[t]{2}{*}{$\mathbf{l}^{2}$} & \multirow{2}{*}{$\begin{array}{l}\text { Nearest coding } \\
\text { genes/cytoband }\end{array}$} & Meta-analysis & \multicolumn{2}{|l|}{$A D$} & GIT Disord \\
\hline & & & & & & & & & $\begin{array}{l}\mathrm{P} \text { - } \\
\text { value }\end{array}$ & $\begin{array}{l}\mathrm{P} \text { - } \\
\text { value }\end{array}$ & $\begin{array}{l}\text { M- } \\
\text { value }\end{array}$ & $\begin{array}{l}\mathrm{P} \text { - } \\
\text { value }\end{array}$ \\
\hline
\end{tabular}

\section{SNPs and loci reaching genome-wide significance after a meta-analysis of AD GWAS and PGM GWAS}

\begin{tabular}{|c|c|c|c|c|c|c|c|c|c|c|c|c|c|c|}
\hline rs2840677 & 1 & rs12058296 & 1 & 66333877 & A & $\mathrm{T}$ & 66.10 & PDE4B/ 1p31.3 & $\begin{array}{l}2.43 \times \\
10^{-8}\end{array}$ & $\begin{array}{l}4.56 \times \\
10^{-8}\end{array}$ & $\begin{array}{l}5.73 \times \\
10^{-3}\end{array}$ & 0.94 & $\begin{array}{l}2.20 \times \\
10^{-7}\end{array}$ & 1 \\
\hline rs6695557 & & & 1 & 66349013 & $A$ & G & 57.16 & PDE4B/ 1p31.3 & $\begin{array}{l}8.46 \times \\
10^{-9}\end{array}$ & $\begin{array}{l}2.08 \times \\
10^{-8}\end{array}$ & $\begin{array}{l}3.02 \times \\
10^{-3}\end{array}$ & 0.97 & $\begin{array}{l}1.89 \times \\
10^{-7}\end{array}$ & 1 \\
\hline rs12058296 & & & 1 & 66402424 & A & C & 84.60 & PDE4B/ 1p31.3 & $\begin{array}{l}5.02 \times \\
10^{-9}\end{array}$ & $\begin{array}{l}9.37 \times \\
10^{-8}\end{array}$ & $\begin{array}{l}3.22 \times \\
10^{-5}\end{array}$ & 0.99 & $\begin{array}{l}8.68 \times \\
10^{-6}\end{array}$ & 1 \\
\hline rs4147104 & 2 & rs4147104 & 1 & 207882194 & $A$ & G & 13.54 & CD46 / 1q32.2 & $\begin{array}{l}5.47 \times \\
10^{-9}\end{array}$ & $\begin{array}{l}1.48 \times \\
10^{-8}\end{array}$ & $\begin{array}{l}1.02 \times \\
10^{-6}\end{array}$ & 1.00 & $\begin{array}{l}6.12 \times \\
10^{-4}\end{array}$ & 1 \\
\hline rs709210 & 3 & rs7642934 & 3 & 50357869 & A & C & 93.99 & $\begin{array}{l}\text { HYAL2 / } \\
\text { 3p21.31 }\end{array}$ & $\begin{array}{l}4.39 \times \\
10^{-8}\end{array}$ & $\begin{array}{l}1.59 \times \\
10^{-7}\end{array}$ & $\begin{array}{l}1.55 \times \\
10^{-2}\end{array}$ & NA & $\begin{array}{l}6.28 \times \\
10^{-8}\end{array}$ & $\uparrow$ \\
\hline rs7642934 & & & 3 & 50174848 & A & G & 93.64 & $\begin{array}{l}\text { SEMA3F / } \\
3 p 21.31\end{array}$ & $\begin{array}{l}2.78 \times \\
10^{-8}\end{array}$ & $\begin{array}{l}1.51 \times \\
10^{-7}\end{array}$ & $\begin{array}{l}7.47 \times \\
10^{-3}\end{array}$ & 0.01 & $\begin{array}{l}8.19 \times \\
10^{-8}\end{array}$ & 1 \\
\hline rs2858331 & 4 & rs2858331 & 6 & 32681277 & G & A & 61.35 & $\begin{array}{l}\text { HLA- } \\
\text { DQA2/ } 6 p 21.32\end{array}$ & $\begin{array}{l}3.08 \times \\
10^{-10}\end{array}$ & $\begin{array}{l}7.14 \times \\
10^{-10}\end{array}$ & $\begin{array}{l}1.24 \times \\
10^{-7}\end{array}$ & 1.00 & $\begin{array}{l}1.18 \times \\
10^{-4}\end{array}$ & 1 \\
\hline rs28895026 & & & 6 & 32391695 & C & $\mathrm{T}$ & 0.00 & $\begin{array}{l}\text { HLA- } \\
\text { DRA/6p21.32 }\end{array}$ & $\begin{array}{l}5.43 \times \\
10^{-9}\end{array}$ & $\begin{array}{l}1.38 \times \\
10^{-8}\end{array}$ & $\begin{array}{l}1.48 \times \\
10^{-7}\end{array}$ & 1.00 & $\begin{array}{l}8.38 \times \\
10^{-3}\end{array}$ & C \\
\hline rs34644948 & 5 & rs34644948 & 16 & 70681658 & $\mathrm{~T}$ & $\mathrm{C}$ & 0.00 & $\begin{array}{l}\text { MTSS2 / } \\
\text { 16q22.1 }\end{array}$ & $\begin{array}{l}2.11 \times \\
10^{-8}\end{array}$ & $\begin{array}{l}5.63 \times \\
10^{-8}\end{array}$ & $\begin{array}{l}1.98 \times \\
10^{-7}\end{array}$ & 1.00 & $\begin{array}{l}3.13 \times \\
10^{-2}\end{array}$ & C \\
\hline rs2584662 & 6 & rs2584662 & 17 & 47470487 & $\mathrm{C}$ & $A$ & 0.00 & PHB / 17q21.33 & $\begin{array}{l}3.94 \times \\
10^{-9}\end{array}$ & $\begin{array}{l}1.00 \times \\
10^{-8}\end{array}$ & $\begin{array}{l}1.98 \times \\
10^{-7}\end{array}$ & 1.00 & $\begin{array}{l}4.91 \times \\
10^{-3}\end{array}$ & C \\
\hline rs11083749 & 7 & rs11083749 & 19 & 45384105 & $\mathrm{~T}$ & C & 0.00 & $\begin{array}{l}\text { APOE / } \\
19 q 13.32\end{array}$ & $\begin{array}{l}2.84 \times \\
10^{-8}\end{array}$ & $\begin{array}{l}7.35 \times \\
10^{-8}\end{array}$ & $\begin{array}{l}1.98 \times \\
10^{-7}\end{array}$ & 1.00 & $\begin{array}{l}3.45 \times \\
10^{-2}\end{array}$ & C \\
\hline
\end{tabular}

\section{SNPs and loci reaching genome-wide significance after a meta-analysis of AD GWAS and GERD GWAS}

\begin{tabular}{|c|c|c|c|c|c|c|c|c|c|c|c|c|c|c|}
\hline rs12058296 & 1 & rs12058296 & 1 & 66402424 & $A$ & $\mathrm{C}$ & 85.75 & PDE4B/ 1p31.3 & $\begin{array}{l}1.05 \times \\
10^{-8}\end{array}$ & $\begin{array}{l}2.10 \times \\
10^{-7}\end{array}$ & $\begin{array}{l}3.22 \times \\
10^{-5}\end{array}$ & 0.99 & $\begin{array}{l}1.74 \times \\
10^{-5}\end{array}$ & 1 \\
\hline rs2503185 & & & 1 & 66461401 & $\mathrm{G}$ & A & 91.93 & PDE4B/ 1p31.3 & $\begin{array}{l}3.44 \times \\
10^{-8}\end{array}$ & $\begin{array}{l}1.21 \times \\
10^{-8}\end{array}$ & $\begin{array}{l}9.53 \times \\
10^{-4}\end{array}$ & 0.25 & $\begin{array}{l}9.60 \times \\
10^{-7}\end{array}$ & 1 \\
\hline rs12561863 & 2 & rs12561863 & 1 & 190897608 & A & $\mathrm{T}$ & 96.02 & BRINP3/ 1q31.1 & $\begin{array}{l}1.68 \times \\
10^{-8}\end{array}$ & $\begin{array}{l}2.89 \times \\
10^{-7}\end{array}$ & $\begin{array}{l}5.76 \times \\
10^{-3}\end{array}$ & 0.00 & $\begin{array}{l}1.05 \times \\
10^{-7}\end{array}$ & 1 \\
\hline rs3774745* & 3 & rs3774745 & 3 & 50204745 & $\mathrm{~T}$ & $\mathrm{C}$ & 92.74 & $\begin{array}{l}\text { SEMA3F/ } \\
\text { 3p21.31* }\end{array}$ & $\begin{array}{l}2.01 \times \\
10^{-9}\end{array}$ & $\begin{array}{l}2.57 \times \\
10^{-9}\end{array}$ & $\begin{array}{l}2.55 \times \\
10^{-4}\end{array}$ & 0.36 & $\begin{array}{l}1.64 \times \\
10^{-7}\end{array}$ & 1 \\
\hline rs28895026 & 4 & rs28895026 & 6 & 32391695 & C & $\mathrm{T}$ & 0.00 & $\begin{array}{l}\text { HLA- } \\
\text { DRA/6p21.32 }\end{array}$ & $\begin{array}{l}2.06 \times \\
10^{-8}\end{array}$ & $\begin{array}{l}5.37 \times \\
10^{-8}\end{array}$ & $\begin{array}{l}1.48 \times \\
10^{-7}\end{array}$ & 1.00 & $\begin{array}{l}4.26 \times \\
10^{-2}\end{array}$ & C \\
\hline rs8067459 & 5 & rs2584662 & 17 & 47444113 & $\mathrm{C}$ & $\mathrm{T}$ & 0.00 & $\begin{array}{l}\text { ZNF652/ } \\
17 q 21.32\end{array}$ & $\begin{array}{l}3.07 \times \\
10^{-8}\end{array}$ & $\begin{array}{l}8.09 \times \\
10^{-8}\end{array}$ & $\begin{array}{l}2.15 \times \\
10^{-7}\end{array}$ & 1.00 & $\begin{array}{l}4.48 \times \\
10^{-2}\end{array}$ & C \\
\hline rs2584662 & & & 17 & 47470487 & C & A & 0.00 & $\mathrm{PHB} / 17 q 21.33$ & $\begin{array}{l}7.72 \times \\
10^{-9}\end{array}$ & $\begin{array}{l}1.96 \times \\
10^{-8}\end{array}$ & $\begin{array}{l}1.54 \times \\
10^{-7}\end{array}$ & 1.00 & $\begin{array}{l}1.02 \times \\
10^{-2}\end{array}$ & C \\
\hline rs11083749 & 6 & rs1132899 & 19 & 45384105 & $\mathrm{~T}$ & C & 0.00 & APOE/ 19q13.32 & $\begin{array}{l}2.63 \times \\
10^{-8}\end{array}$ & $\begin{array}{l}6.88 \times \\
10^{-8}\end{array}$ & $\begin{array}{l}2.46 \times \\
10^{-7}\end{array}$ & 1.00 & $\begin{array}{l}3.14 \times \\
10^{-2}\end{array}$ & C \\
\hline rs1132899 & & & 19 & 45448036 & $\mathrm{~T}$ & C & 94.99 & $\begin{array}{l}\text { APOC2/ } \\
19 q 13.32\end{array}$ & $\begin{array}{l}1.19 \times \\
10^{-8}\end{array}$ & $\begin{array}{l}1.57 \times \\
10^{-7}\end{array}$ & $\begin{array}{l}5.41 \times \\
10^{-8}\end{array}$ & 1.00 & $\begin{array}{l}5.53 \times \\
10^{-3}\end{array}$ & C \\
\hline rs117501883 & 7 & rs117501883 & 19 & 45841296 & $A$ & G & 0.00 & KLC3/ 19q13.32 & $\begin{array}{l}8.96 \times \\
10^{-9}\end{array}$ & $\begin{array}{l}2.32 \times \\
10^{-8}\end{array}$ & $\begin{array}{l}7.13 \times \\
10^{-8}\end{array}$ & 1.00 & $\begin{array}{l}3.78 \times \\
10^{-2}\end{array}$ & $\mathrm{C}$ \\
\hline rs76692930 & & & 19 & 45875851 & $\mathrm{~T}$ & $\mathrm{C}$ & 50.22 & $\begin{array}{l}\text { ERCC2/ } \\
19 q 13.32\end{array}$ & $\begin{array}{l}3.51 \times \\
10^{-8}\end{array}$ & $\begin{array}{l}8.60 \times \\
10^{-8}\end{array}$ & $\begin{array}{l}3.18 \times \\
10^{-6}\end{array}$ & 1.00 & $\begin{array}{l}8.32 \times \\
10^{-4}\end{array}$ & $\mathrm{C}$ \\
\hline \multicolumn{15}{|c|}{ SNPs and loci reaching genome-wide significance after a meta-analysis of AD GWAS and PUD GWAS } \\
\hline rs36133610 & 1 & rs36133610 & 2 & 234067884 & A & G & 0.00 & INPP5D/ 2q37.1 & $\begin{array}{l}1.24 \times \\
10^{-8}\end{array}$ & $\begin{array}{l}3.41 \times \\
10^{-8}\end{array}$ & $\begin{array}{l}5.85 \times \\
10^{-8}\end{array}$ & 1.00 & $\begin{array}{l}4.90 \times \\
10^{-2}\end{array}$ & C \\
\hline
\end{tabular}




\begin{tabular}{|c|c|c|c|c|c|c|c|c|c|c|c|c|c|c|}
\hline rs9270599 & 2 & rs9270599 & 6 & 32561656 & G & $A$ & 26.29 & $\begin{array}{l}\text { HLA- } \\
\text { DRA/6p21.32 }\end{array}$ & $\begin{array}{l}9.12 \times \\
10^{-9}\end{array}$ & $\begin{array}{l}2.59 \times \\
10^{-8}\end{array}$ & $\begin{array}{l}5.60 \times \\
10^{-8}\end{array}$ & 1.00 & $\begin{array}{l}2.72 \times \\
10^{-2}\end{array}$ & C \\
\hline rs530324 & 3 & rs530324 & 8 & 27491186 & $\mathrm{C}$ & $\mathrm{G}$ & 76.92 & SCARA3/ 8p21.1 & $\begin{array}{l}2.27 \times \\
10^{-8}\end{array}$ & $\begin{array}{l}6.17 x \\
10^{-8}\end{array}$ & $\begin{array}{l}3.32 \times \\
10^{-7}\end{array}$ & 1.00 & $\begin{array}{l}2.00 \times \\
10^{-3}\end{array}$ & C \\
\hline rs73976310 & 4 & rs73976310 & 17 & 5014212 & $A$ & G & 31.11 & USP6/ 17p13.2 & $\begin{array}{l}1.20 \times \\
10^{-8}\end{array}$ & $\begin{array}{l}3.67 \times \\
10^{-8}\end{array}$ & $\begin{array}{l}7.04 \times \\
10^{-8}\end{array}$ & 1.00 & $\begin{array}{l}2.70 \times \\
10^{-2}\end{array}$ & C \\
\hline rs28363848 & 5 & rs28363848 & 19 & 45257201 & $\mathrm{~T}$ & $\mathrm{G}$ & 41.06 & BCL3/ 19q13.32 & $\begin{array}{l}1.04 \times \\
10^{-8}\end{array}$ & $\begin{array}{l}2.96 \times \\
10^{-8}\end{array}$ & $\begin{array}{l}5.63 \times \\
10^{-8}\end{array}$ & 1.00 & $\begin{array}{l}2.60 \times \\
10^{-2}\end{array}$ & C \\
\hline rs3852865 & 6 & rs3852865 & 19 & 51714065 & $A$ & G & 58.20 & CD33/ 19q13.41 & $\begin{array}{l}1.81 \times \\
10^{-8}\end{array}$ & $\begin{array}{l}5.19 \times \\
10^{-8}\end{array}$ & $\begin{array}{l}1.63 \times \\
10^{-7}\end{array}$ & 1.00 & $\begin{array}{l}9.90 \times \\
10^{-3}\end{array}$ & C \\
\hline rs7245846 & & & 19 & 51731176 & $A$ & G & 5.22 & CD33/ 19q13.41 & $\begin{array}{l}2.32 \times \\
10^{-8}\end{array}$ & $\begin{array}{l}6.71 \times \\
10^{-8}\end{array}$ & $\begin{array}{l}1.19 \times \\
10^{-7}\end{array}$ & 1.00 & $\begin{array}{l}4.00 \times \\
10^{-2}\end{array}$ & C \\
\hline
\end{tabular}

SNP: Single nucleotide polymorphism; Chr: Chromosome; EA: Effect allele; NEA: Non-effect allele; I2: I-square for heterogeneity assessment; Se: Standard error; P: P-value; Meta-analysis model use was RE2C and RE2. RE2C: GWAS meta-analysis methods that account for sample overlap and heterogeneity. RE2: adjusts for heterogeneity. *The m-value suggests that the SNP rs3774745 has an effect on GERD but is ambiguous for AD. Comparing the results with results of $\mathrm{BE}$, and P-value, the locus is more likely to influence both traits.

Table 5: Summary of MR analysis results for AD and GIT disorders

\begin{tabular}{|c|c|c|c|c|c|c|c|c|c|c|c|c|c|c|}
\hline \multirow[t]{2}{*}{$\begin{array}{l}\text { Exposure } \\
\text { (nSNPs) }\end{array}$} & \multirow[t]{2}{*}{ Outcome } & \multicolumn{2}{|l|}{ IVW } & \multicolumn{2}{|c|}{$\begin{array}{l}\text { Weighted } \\
\text { median }\end{array}$} & \multicolumn{2}{|c|}{ MR-Egger } & \multicolumn{5}{|c|}{ MR-PRESSO } & \multicolumn{2}{|l|}{$\begin{array}{l}\text { MR-Egger } \\
\text { Intercept }\end{array}$} \\
\hline & & Beta & $\mathbf{P}$ & Beta & $P$ & Beta & $\mathbf{P}$ & $\begin{array}{l}\text { Global } \\
\text { test P }\end{array}$ & $\begin{array}{l}\text { Raw } \\
\text { Beta }\end{array}$ & $\mathbf{P}$ & $\begin{array}{l}\text { Corrected } \\
\text { Beta }\end{array}$ & $\mathbf{P}$ & Intercept & $P$ \\
\hline$A D(28)$ & GERD & -0.053 & 0.266 & 0.011 & 0.860 & -0.059 & 0.362 & 0.113 & -0.052 & 0.276 & - & - & 0.00034 & 0.87 \\
\hline GERD (24) & $A D$ & 0.014 & 0.351 & -0.002 & 0.920 & -0.053 & 0.597 & 0.435 & 0.0136 & 0.361 & - & - & 0.0025 & $0.5 \mathrm{C}$ \\
\hline$A D(28)$ & PGM & -0.061 & 0.112 & -0.016 & 0.769 & -0.045 & 0.391 & 0.231 & -0.061 & 0.123 & - & - & -0.001 & 0.63 \\
\hline PGM (17) & $A D$ & 0.023 & 0.322 & -0.005 & 0.837 & -0.148 & 0.199 & 0.017 & 0.023 & 0.337 & 0.009 & 0.661 & 0.007 & 0.13 \\
\hline AD (28) & PUD & 0.036 & 0.651 & 0.144 & 0.211 & 0.071 & 0.504 & 0.113 & 0.036 & 0.60 & - & - & -0.002 & 0.61 \\
\hline PUD (8) & $A D$ & 0.021 & 0.238 & 0.025 & 0.122 & 0.055 & 0.658 & 0.0104 & 0.021 & 0.277 & 0.0331 & 0.053 & -0.00291 & 0.77 \\
\hline$A D(28)$ & Diverticular & -0.055 & 0.597 & -0.214 & 0.105 & -0.12 & 0.397 & 0.094 & -0.055 & 0.601 & - & - & 0.0034 & $0.4 \varepsilon$ \\
\hline $\begin{array}{l}\text { Diverticular } \\
\text { (16) }\end{array}$ & $A D$ & -0.001 & 0.883 & -0.001 & 0.905 & 0.007 & 0.811 & 0.316 & -0.001 & 0.884 & - & - & -0.00076 & 0.77 \\
\hline$A D(28)$ & IBS* & 0.043 & 0.623 & 0.142 & 0.123 & -0.016 & 0.888 & 0.0012 & 0.043 & 0.626 & 0.010 & 0.892 & 0.0032 & 0.43 \\
\hline AD (28) & Gastritis-D* & -0.085 & 0.267 & -0.101 & 0.273 & -0.173 & 0.098 & 0.034 & -0.085 & 0.277 & - & - & 0.0046 & $0.1 \mathrm{C}$ \\
\hline$A D(28)$ & IBD & 0.254 & 0.104 & 0.365 & 0.094 & 0.277 & 0.231 & 0.327 & 0.254 & 0.115 & - & - & -0.00097 & $0.8 \varepsilon$ \\
\hline IBD (24) & $A D$ & -0.0005 & 0.895 & -0.003 & 0.526 & 0.004 & 0.607 & 0.316 & -0.0005 & 0.895 & - & - & 0.00073 & $0.4 \mathrm{C}$ \\
\hline
\end{tabular}

nSNP: number of SNPs utilised as instrumental variables, SNP: single nucleotide polymorphism, AD: Alzheimer's disease, GERD: gastroesophageal reflux disease, PUD: peptic ulcer disease, PGM: medications for GERD or PUD, Diverticular: diverticular disease, IBS: irritable bowel syndrome, IBD: inflammatory bowel disease, IVW: inverse variance weighted, P: P-value, MR-PRESSO: Mendelian Randomization Pleiotropy RESidual Sum and Outlier, Gastritis-D: Gastritisduodenitis, * Only one genome-wide significant SNPs available for IBS, and 3 for Gastritis-D, so we are unable to carry out MR using the traits as the exposure variable. Note spaces marked with a dash indicate that there were no outlier SNPs and hence there were no outlier corrected results in the MR-PRESSO analysis.

Table 6: Partial causality assessment using the Latent Causal Variable approach 


\begin{tabular}{|c|c|c|c|c|c|c|}
\hline Trait 1 & Trait 2 & GCP & SE & P & $r_{g}$ & $r_{g}-P$ \\
\hline \multirow[t]{8}{*}{$A D$} & Gastritis-duodenitis (Main ICD10: K29) & -0.69 & 0.27 & 0.0026 & 0.39 & 0.033 \\
\hline & Gastritis-duodenitis (PheCode_535_SAIGE) & -0.36 & 0.39 & 0.43 & 0.25 & 0.018 \\
\hline & PGM & -0.45 & 0.37 & 0.22 & 0.21 & 0.0064 \\
\hline & PUD & 0.49 & 0.32 & 0.24 & 0.33 & 0.0077 \\
\hline & IBS & 0.35 & 0.29 & 0.38 & 0.19 & 0.043 \\
\hline & GERD & -0.01 & 0.58 & 0.64 & 0.25 & 0.060 \\
\hline & Diverticular disease (Main ICD10: K57) & 0.23 & 0.10 & 0.000272 & 0.26 & 0.0396 \\
\hline & Lansoprazole & -0.38 & 0.17 & 0.001129 & 0.43 & 0.0176 \\
\hline
\end{tabular}

AD: Alzheimer's disease, GCP: Genetic causal proportion, SE: Standard error, P: P-value, $r_{\mathrm{g}}$ : genetic correlation, rg-P: genetic correlation P-value, GERD: gastroesophageal reflux disease, PUD: peptic ulcer disease, PGM: GWAS for medication use in GERD and PUD.

\section{Figures}

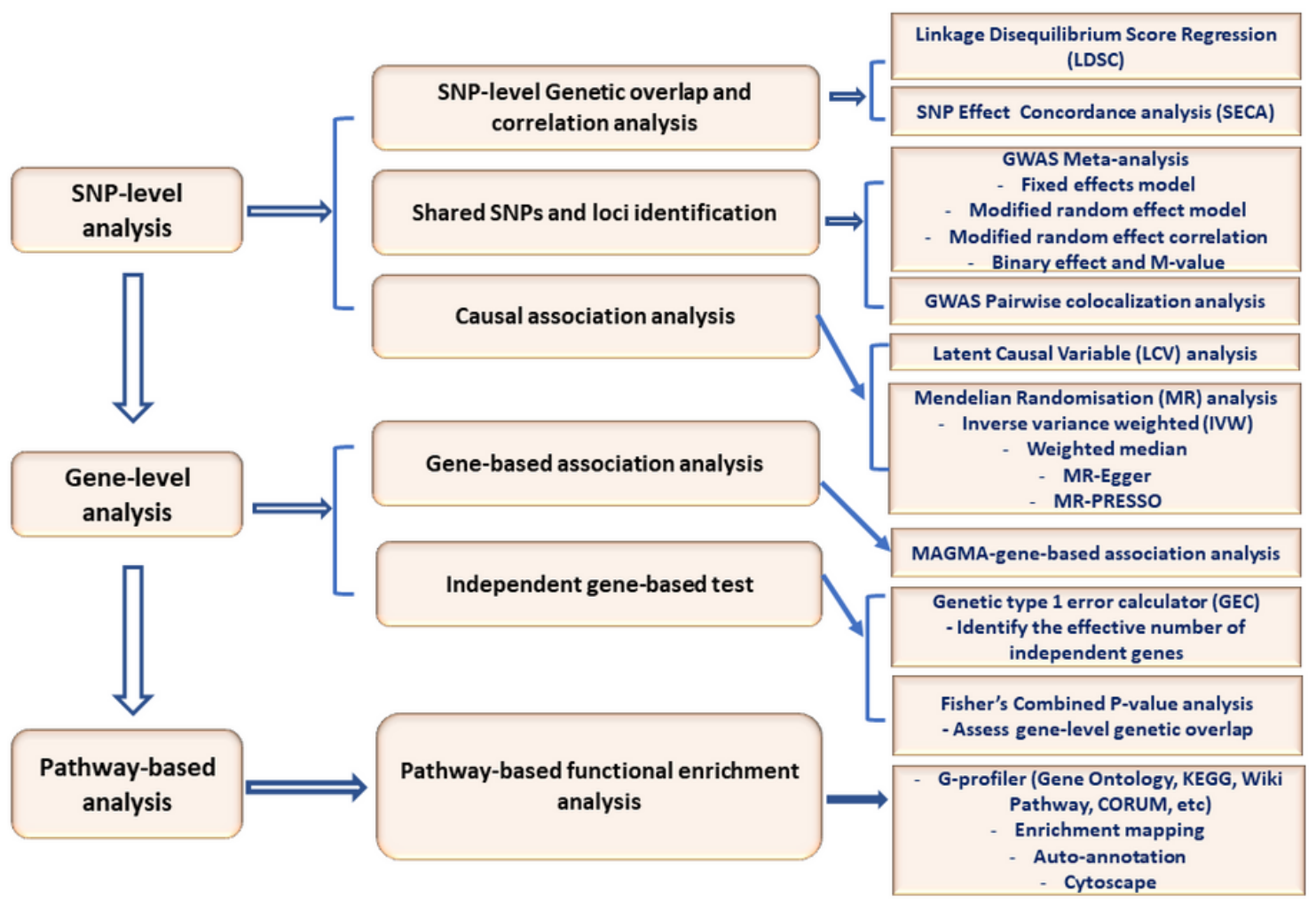

Figure 1

Fig. 1 presents a schematic workflow and design for this study. Briefly, we performed three broad levels of analyses-SNP-level, gene-level, and pathway-based analysis-to comprehensively assess the genetic relationships between AD and GIT disorders. In each of the levels, we analysed well-powered GWAS data using a set of well-regarded statistical genetics methods. First, we used the 'SNP effect concordance analysis' (SECA) [28] method for SNP-level genetic overlap assessment and the linkage disequilibrium score regression (LDSC) [29] method for genetic correlation analysis between AD and each of the GIT traits. Second, to identify SNPs and susceptibility loci shared by AD and GIT disorders, we carried out GWAS meta-analysis using several complementary models, leveraging the increased power from data pooling and pleiotropy of genetic variants. We also applied the pairwise GWAS (colocalisation) method [30] to identify independent genomic loci with shared genetic influence on AD and GIT disorders. Third, using the Mendelian randomisation (MR) [31] and the Latent Causal Variable (LCV) [32] methods, we investigated potential causal (or partial causal [LCV]) associations between AD and each of the GIT disorders. Fourth, we performed gene-based association analysis to identify genes shared by AD and GIT disorders reaching genome-wide significance. Lastly, we used pathway-based analysis to identify potential biological mechanisms shared by AD and GIT disorders. 


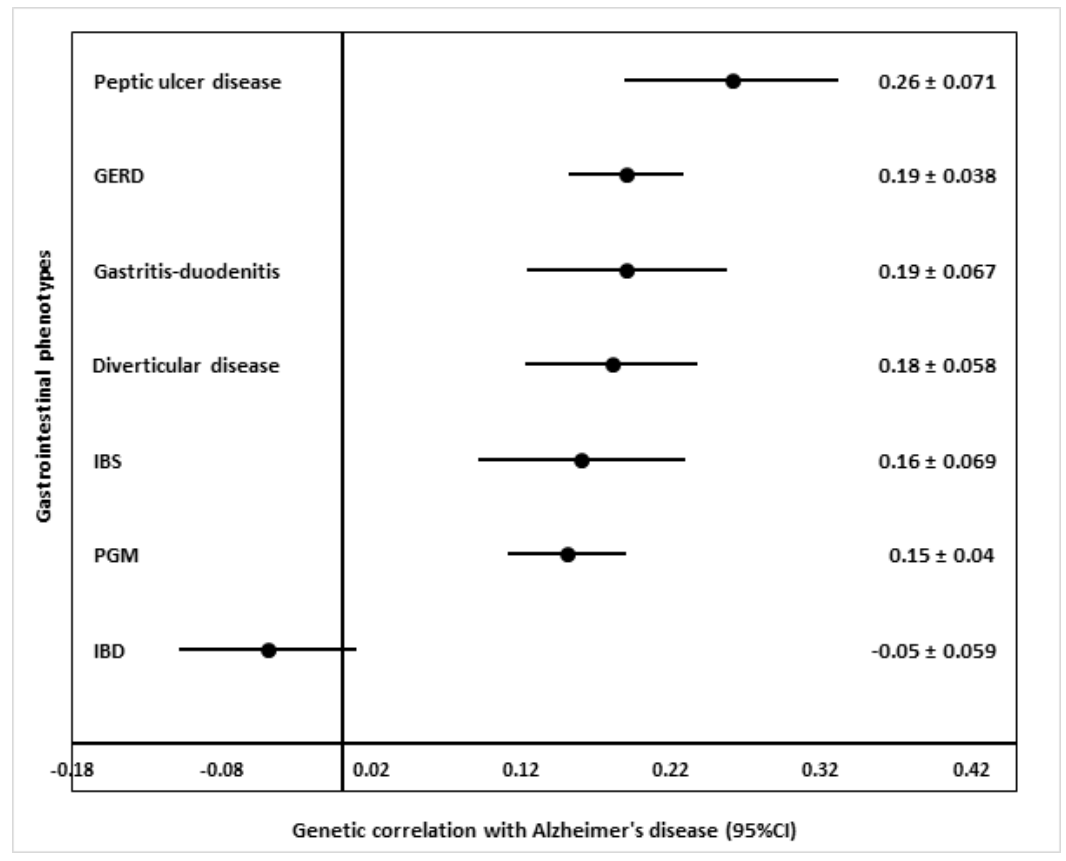

\section{Figure 2}

Genetic correlation between Alzheimer's disease and gastrointestinal traits without the APOE and MHC regions GERD: gastroesophageal reflux disease, IBS: irritable bowel syndrome, PGM: medications use for peptic ulcer disease and GERD, IBD: inflammatory bowel disease. Genetic correlation analysis was conducted using the Linkage disequilibrium score regression analysis method. In all the analyses, the genetic covariance intercept was not constrained

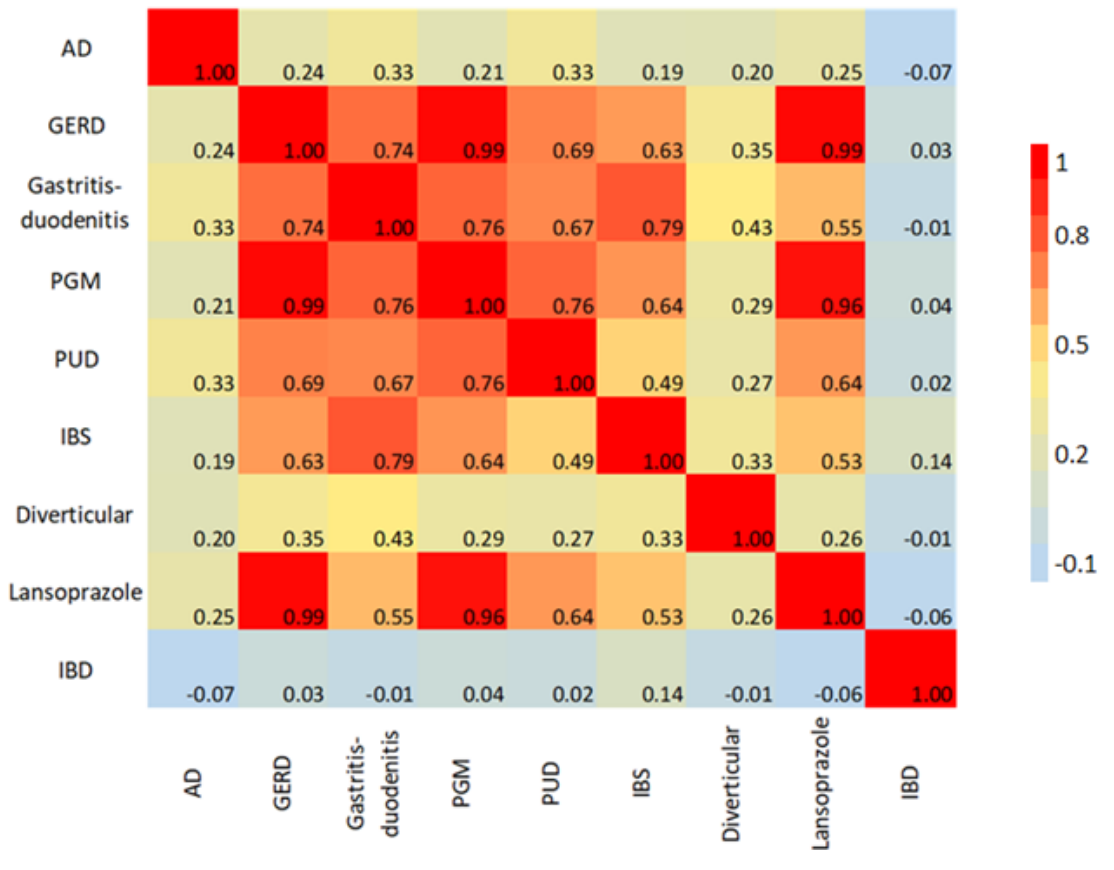

\section{Figure 3}

Heatmap of genetic correlation between GWAS summary statistics analysed in this study. AD: Alzheimer's disease, GERD: Gastroesophageal reflux disease, PUD: Peptic ulcer disease, PGM: medications for PUD and GERD, IBS: Irritable bowel syndrome, IBD: Inflammatory bowel disease. The genetic correlation was estimated using the Linkage disequilibrium score regression (LDSC) analysis software. 
a. Gene Ontology: Biological Pathway

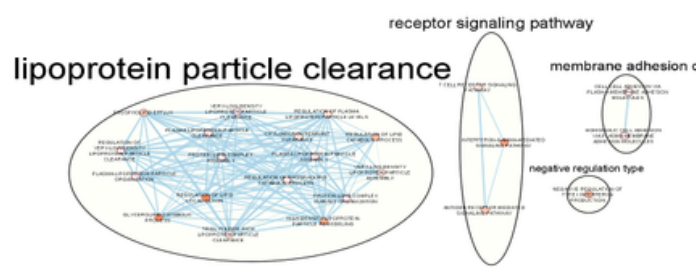

b. Gene Ontology: Cellular Components

side membrane vesicle

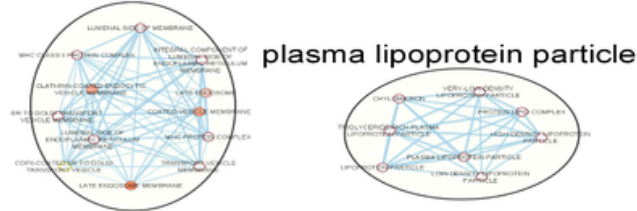

c. Gene Ontology: Molecular Function

\section{peptide antigen binding}

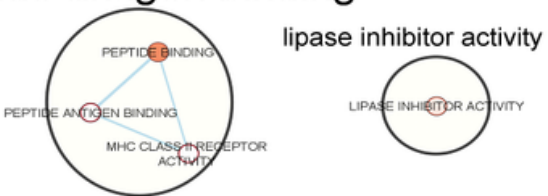

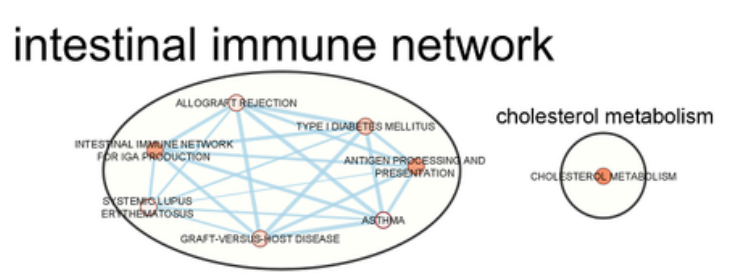

e. Reactome, Wiki pathway and Transcription Factor Binding site

assembly clearance plasma

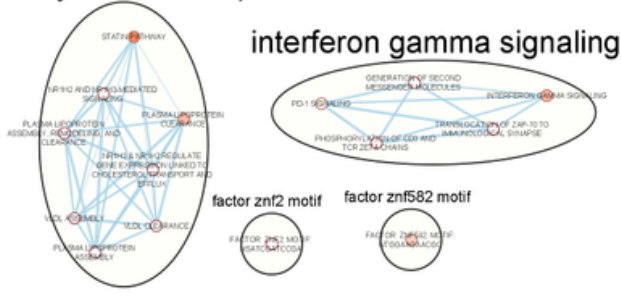

Figure 4

Clusters of significantly enriched biological pathways for AD and GERD

\section{Supplementary Files}

This is a list of supplementary files associated with this preprint. Click to download.

- SupplementaryNotes.docx

- SupplementaryTablesADGITdisordermanuscript.xIsx 